University of Wollongong

Research Online

Australian Institute for Innovative Materials -

Papers

Australian Institute for Innovative Materials

$1-1-2013$

\title{
Flexible Supercapacitors - Development of Bendable Carbon Architectures
}

Zhiqiang Niu

Nanyang Technological University, Singapore

Lili Liu

Nanyang Technological University, Singapore

Peter C. Sherrell

University of Wollongong, psherrel@uow.edu.au

Jun Chen

University of Wollongong, junc@uow.edu.au

Xiaodong Chen

Nanyang Technological University, Singapore

Follow this and additional works at: https://ro.uow.edu.au/aiimpapers

Part of the Engineering Commons, and the Physical Sciences and Mathematics Commons

Research Online is the open access institutional repository for the University of Wollongong. For further information contact the UOW Library: research-pubs@uow.edu.au 


\title{
Flexible Supercapacitors - Development of Bendable Carbon Architectures
}

\author{
Abstract \\ As energy storage devices, Supercapacitors (SCs), also known as electrochemical capacitors, possess \\ high power densities, excellent reversibility and long cycle life. SCs could be applied to diverse fields \\ including electric vehicles, pulse power applications and portable devices. Flexible SCs have attracted \\ significant attention for powering recently developed portable, flexible and wearable electronics. During \\ the past several years, a variety of bendable carbon-based electrode architechtures and flexible SC \\ devices with different designs have been successfully prepared. In this review, we will describe recent \\ developments in the preparation of such bendable carbon-based electrode architechtures and the \\ subsequent design of flexible SC devices. Future development and prospects of flexible SCs are also \\ discussed. \\ Keywords \\ architectures, supercapacitors, carbon, bendable, flexible, development \\ Disciplines \\ Engineering | Physical Sciences and Mathematics \\ Publication Details \\ Niu, Z., Liu, L., Sherrell, P. C., Chen, J. and Chen, X. (2013). Flexible Supercapacitors - Development of \\ Bendable Carbon Architectures. In Y. Hu, U. Burghaus and S. Qiao (Eds.), Nanotechnology for Sustainable \\ Energy (pp. 101-141). United States of America: American Chemical Society.
}




\section{RESERVE THIS SPACE}

Flexible Supercapacitors - Development of Bendable Carbon Architectures

By Zhiqiang Niu, Lili Liu, Peter Sherrell, Jun Chen* and Xiaodong Chen*

[*] Prof. X. D. Chen, Dr. Z. Q. Niu, Dr. L. L. Liu

School of Materials Science and Engineering, Nanyang

Technological University, 50

Nanyang Avenue, Singapore 639798

E-mail: chenxd@ntu.edu.sg

Website: http://www.ntu.edu.sg/home/chenxd/

[*] Associate Prof. Jun Chen, Dr Peter Sherrell

Intelligent Polymer Research Institute, ARC Centre of Excellence for Electromaterials Science, Australian Institute of Innovative Materials, Innovation Campus, University of Wollongong, Northfields Avenue, Wollongong, NSW 2522, Australia E-mail: junc@uow.edu.au

Keywords: Supercapacitors; carbon; flexible

As energy storage devices, Supercapacitors (SCs), also known as electrochemical capacitors, possess high power densities, excellent reversibility and long cycle life. SCs could be applied to diverse fields including electric vehicles, pulse power applications and portable devices. Flexible SCs have attracted significant attention for powering recently developed portable, flexible and wearable electronics. During the past several years, a variety of bendable

\section{RESERVE THIS SPACE}


carbon-based electrode architechtures and flexible SC devices with different designs have been successfully prepared. In this review, we will describe recent developments in the preparation of such bendable carbon-based electrode architechtures and the subsequent design of flexible SC devices. Future development and prospects of flexible SCs are also discussed.

\section{Introduction}

As energy storage devices, Supercapacitors (SCs), also known as electrochemical capacitors, possess high power densities, excellent reversibility and long cycle life. ${ }^{[1-4]}$ SCs could be applied to diverse fields such as electric vehicles, pulse power applications and portable devices. ${ }^{[1-2]}$ Generally, on the basis of the energy storage mechanism, SCs can be classified into two categories. ${ }^{[1]}$ One is the electrical double layer capacitor (EDLC), wherein the capacitance is ascribed to the pure electrostatic charge accumulated at the electrode/electrolyte interface. This charge is strongly dependent on the ability of electrolyte ions to access the surface area of the electrode materials. The other category is the pseudo-capacitor, where fast and reversible faradic processes occur due to electro-active species.

Figure 1 depicts the typical structure of a conventional SC. A conventional SC consists of four main components, including current collector, electrode materials, separator, and electrolyte, which are packaged together. In contrast to batteries, most SCs use the same materials in both electrodes (positive and negative). Carbon-based materials such as activated carbons (ACs), CNTs (CNTs), graphene and graphene oxide (GO) derivatives, and carbon aerogels (CAGs) are the most widely used electrodes because of their accessibility, ease of processing, relatively low cost, non-toxicity, high chemical stability, and wide temperature range. ${ }^{[1]}$ According to the storage mechanism, pure carbonbased SCs are typically EDLCs, which depend on the specific surface area 
(SSA) of carbon-based electrodes. However, the major problem of high SSA carbon is that not all the BET surface area is electrochemically accessible when in contact with electrolyte, such that the gravimetric capacitance of various carbon materials does not increase linearly with the SSA, resulting in a low energy density. ${ }^{[5]}$

To improve their energy density, the pseudo-capacitance from quick and reversible faradic reactions such as electrosorption or from the oxidationreduction of electroactive materials is often coupled with the EDLC to enhance the overall capacitance of the electrode materials. Conductive polymers (CP), such as polypyrrole (PPy) or polyaniline (PANI), and metal oxides (MOs), are the most commonly studied species which provide pseudo-capacitance when combined with a carbon support material. ${ }^{[2]}$ The doped carbon electrodes display high energy density due to pseudo-capacitance originating from the CP or MO. However, their power density is dramatically reduced when compared to the pure carbon-based electrodes, due to the relatively poor electrical conductivity of CPs and MOs. Therefore, one of the most critical aspects in the development of carbon materials doped by CP or/and MOs is to optimize the energy density without causing a deterioration in their high power capability as the balance of these two parameters determine the ultimate performance of the SCs.

Energy storage systems are moving towards light, thin, low-cost, environmentally friendly, high-performance and often flexible devices due to the urgent demand of portable, flexible and wearable electronics. ${ }^{[6]}$ As such, developing high power, high energy, flexible SCs has attracted significant attention. CNTs and graphene possess high conductivity, SSA, and mechanical properties, which endow then with the capability of constructing flexible electrodes. In addition, in traditional SC devices, metallic foils or foams are used as current collectors for both the anode and cathode due to the poor conductivity of the active electrode materials. However, the use of such metallic current collectors add significant mass to the SC, resulting in devices that are too 
heavy, bulky or inflexible, limiting their use in applications where space and weight are constraints. Therefore, conventional SC configurations cannot meet the demand of flexible devices, and novel SC configurations with simplified designs and high flexibility should be considered. In the past several years, considerable attention has been paid to the fabrication of various forms of flexible SC electrodes and devices based on CNT and/or graphene. In this review, we will describe recent development in the preparation of bendable carbon architectures and the design of flexible SCs based on these electrodes. The further developments and prospects of flexible SCs in this exciting field are also discussed.

\section{Carbon materials-based flexible EDLCs}

\subsection{CNTs-based flexible EDLCs}

Flexible EDLCs rely on large-surface-area, conductive electrodes with strong mechanical properties. CNTs, having a high electroactive surface area combined with high conductivity and durable mechanical properties, are attractive candidates for electrodes in flexible SC devices. Following the successful synthesis of CNT films, flexible CNT electrodes became significantly more viable.

Niu et al pioneered in fabricating the flexible multi-walled CNT (MWCNT) electrodes via three steps: 1) disassembling the MWCNT aggregates and introducing chemical functional groups on the surface of the MWCNTs by nitric acid treatment; 2) dispersing the functionalized hydrophilic nanotubes in water; 3) reassembling the individualized CNTs into an interconnected, entangled, freestanding film by filtration. ${ }^{[7]}$ These MWCNT film electrodes have smooth surfaces, uniform thicknesses, acceptable mechanical strength and low electronic resistance $\left(1.6 \times 10^{-2} \Omega\right)$. They are flexible and can be easily bent, rolled, and cut into different shapes and sizes. The measured density of the 
electrode is $0.8 \mathrm{~g} / \mathrm{cc}$, which can be controlled by varying the conditions of the nitric acid oxidation. Total pore volume is $0.79 \mathrm{cc} / \mathrm{g}$ with the micro-pore $(<20$ $\AA$ ) volume being negligible at $0.0016 \mathrm{cc} / \mathrm{g}$. The average pore diameter is $92 \AA$, which is accessible to the electrolyte. The specific surface area of these MWCNT films is $430 \mathrm{~m}^{2} / \mathrm{g}$. The combination of porous structure and high conductivity in the MWCNT film electrodes results in excellent electrochemical behaviour. The specific capacitance and power density of the MWCNT film electrodes are $104 \mathrm{~F} / \mathrm{g}$ and $8 \mathrm{~kW} / \mathrm{kg}$ respectively. The knee frequency is usually used to evaluate the frequency dependence of a capacitor and considered to be the critical frequency where supercapacitors begin to exhibit capacitive behavior. At a frequency higher than the "knee", the real part of the impedance is frequency dependent and very small capacitance will be obtained. At a frequency below this value, the resistance changes weakly with frequency and the capacitor behavior tends to approach that of a pure capacitance. The knee frequency of most commercially available supercapacitors is less than $1 \mathrm{~Hz}$. The knee frequency of such MWCNT electrodes is up to about $100 \mathrm{~Hz}$. A very high "knee" frequency of $100 \mathrm{~Hz}$ suggests that most of the stored energy of the capacitor is accessible at frequencies as high as $100 \mathrm{~Hz}$.

Similarly, by filtering DNA-dispersed double-walled CNTs (DWCNTs) into a thin film and thermally treating the film in argon, freestanding, thin, and bendable electrodes for SCs were fabricated. ${ }^{[8]}$ The DNA wrapped around the DWCNTs was converted to phosphorus-enriched carbon during thermal treatment of the film under an argon atmosphere, giving rise to strong redox peaks at around $0.4 \mathrm{~V}$. The combination of the large capacitance $(66.9 \mathrm{~F} / \mathrm{g})$ from the DNA-derived carbons and the high electrical conductivity of CNTs allow DWNT/DNA films to be used as a potential flexible electrode material for SCs.

Based on flexible polyethylene-terephthalate (PET) substrate, Cui and coworkers successfully prepared flexible single-walled CNT (SWCNT) film electrodes by utilizing spray coating technology, serving as both electrodes and 
charge collectors, as shown in Figure 2a and b. ${ }^{[6]}$ The demonstrated high current carrying capability together with the substantial mechanical strength of the SWCNT networks assists the creation of flexible robust devices. The use of solid gel or polymer electrolytes opens up automatable fabrication avenues for SCs, including printing. The use of a liquid electrolyte in an electrochemical device requires both robust encapsulation to prevent leakage and a separator to avoid electrical contact between the electrodes. Solid gel or polymer electrolytes offer dual functionality as they combine the separator and the electrolyte into a single layer. It also avoids potential leakages since the electrolyte is bound within the polymer matrix. The specific capacitance of SWCNT thin film supercpacitors obtained using the polymer electrolyte was about $110 \mathrm{~F} / \mathrm{g}$, which is comparable to examined aqueous electrolytes. The SCs also show very high energy $(6 \mathrm{Wh} / \mathrm{kg})$ and power densities $(23 \mathrm{~kW} / \mathrm{kg})$. The simplified architecture and the sole use of printable materials may lead to a new class of entirely printable charge storage devices, allowing for full integration with the emerging field of printed electronics. In addition, this group demonstrates that commercially available paper can also be made highly conductive with a sheet resistance ( $1 \Omega /$ square) by using simple solution processes to achieve conformal coating of SWCNT and silver nanowire films, as shown in Figure 2c-h. ${ }^{[9]}$ Compared with plastics, paper substrates can remarkably improve film adhesion, simplify the coating process, and significantly lower the device cost. The combination of the flexibility of individual CNTs, the strong binding of the CNTs with the paper fibers, and the porous morphology of the paper endow the CNT-conductive paper with excellent mechanical properties (Figure 2g). The conductive paper with CNT thicknesses from $100 \mathrm{~nm}$ to $5 \mu \mathrm{m}$ can be folded without any measurable change in electrical conductivity. Fatigue tests show that the conductive paper can be bent to a $2-\mathrm{mm}$ radius 100 times with a resistance increase of less than $5 \%$. Such flexibility could meet the demand of flexible SC electrodes, including various rolled-up devices. The SCs, in which 
the CNT-conductive paper acts as both the active electrode and current collector, show excellent electrochemical properties. A specific capacitance of $200 \mathrm{~F} / \mathrm{g}$ (Figure $2 \mathrm{~h}$ ), a specific energy of 30-47 Wh/kg, a specific power of $200 \mathrm{~kW} / \mathrm{kg}$ (considering only the mass of CNT), and a stable cycle life of over 40,000 cycles are achieved for a liquid electrolyte-based CNT-conductive paper SC. To make the SCs more compact and flexible, all-solid SCs based on CNTconductive paper were prepared by using an ionic-liquid-based gel electrolyte. ${ }^{[10]}$ The specific capacitance of the all-solid-state $\mathrm{SC}$ was $135 \mathrm{~F} / \mathrm{g}$ at a current density of $2 \mathrm{~A} / \mathrm{g}$ (considering only the mass of active materials). The maximum power and energy densities of the all-solid-state SC were $164 \mathrm{~kW} / \mathrm{kg}$ and $41 \mathrm{Wh} / \mathrm{kg}$, respectively. More importantly, the all-solid-state SC was flexible, and could be repeatedly bent without any significant variation in the electrochemical properties including specific capacitance, power and energy density $(<5 \%)$.

Apart from PET and commercially available paper, textiles such as cotton or polyester are also excellent support materials for the deposition of CNTs and nanowires, due to the flexibility and porosity inherent in textile fabrication techniques, such as weaving or pressing. Cui et al fabricated conductive textile through an extremely simple "dipping and drying" process using a SWCNT ink. ${ }^{[11]}$ The electrical conductivity of these conductive textiles improved from $\sim 5$ to $\sim 125 \mathrm{~S} / \mathrm{cm}$ with the decrease of the thickness of the cotton sheets from $\sim 2$ $\mathrm{mm}$ to $\sim 80 \mu \mathrm{m}$ under mechanical pressing. The sheet resistance of conductive textiles was less than $1 \Omega$ /square. The conductive textiles show outstanding mechanical properties, strong binding between SWCNTs and the textile, flexibility, and stretchability, making them an ideal candidate for flexible, stretchable SCs. SCs made from these conductive textiles show a high area capacitance, up to $0.48 \mathrm{~F} / \mathrm{cm}^{2}$, and high specific energy of $20 \mathrm{Wh} / \mathrm{kg}$ at a specific power of $10 \mathrm{~kW} / \mathrm{kg}$. These highly conductive textiles can provide new design opportunities for wearable electronics and energy storage applications. Based on 
the conductive cotton paper coated with SWCNTs, flexible solid-state SCs can be obtained by using poly (vinyl alcohol) (PVA)/phosphoric acid as the electrolyte. ${ }^{[12]}$ The as-prepared SC is very flexible and can be bent by more than $90^{\circ}$ without showing any fracture. The $\mathrm{SC}$ is also very thin with a thickness of only $1.3 \mathrm{~mm}$. The specific capacitance of the as-fabricated SC was $115.83 \mathrm{~F} / \mathrm{g}$, and specific energy was $48.86 \mathrm{Wh} / \mathrm{kg}$.

Integrated, flexible SC structures containing the three essential components (electrodes, spacer, and electrolyte) of the electrochemical device enable SCs to be embedded as various functional devices in a wide range of innovative products such as smart cards, displays, and implantable medical devices. By combining a CNT array, cellulose and a room temperature ionic liquid (RTIL), Ajayan et al integrated electrodes, spacer, and electrolyte into one single contiguous nanocomposite unit that can serve as a building block for a variety of mechanically flexible thin energy storage devices. ${ }^{[13]}$ The units are used to build various flexible $\mathrm{SCs}$, batteries and hybrid dual-storage battery-in-SC devices. A specific capacitance of $22 \mathrm{~F} / \mathrm{g}$ and a power density of $1.5 \mathrm{~kW} / \mathrm{kg}$ were obtained at room temperature for the nanocomposite RTIL SC.

Freestanding and highly conductive SWCNT films with a continuous reticulate architecture were fabricated by improving the floating catalyst chemical vapor deposition technique. ${ }^{[14-15]}$ Such films are different from conventional SWCNT films prepared by other post-deposition methods that produce short and randomly-oriented structures. A continuous reticulate architecture endows directly synthesized films superior electrical conductivity (about $2000 \mathrm{~S} / \mathrm{cm}$ ) and better mechanical properties than that of post-deposited SWCNT films. Based on our directly synthesized SWCNT films (Figure 3b and c), we assembled compact-designed SCs using large-scaled free-standing and flexible SWCNT films as both anode and cathode, as shown in Figure 3. A prototype of the processing procedures was first developed to obtain uniform spreading of the SWCNT films onto the separators, serving as both electrodes 
and charge collectors, allowing for a device without metallic current collectors (Figure 3a and d), leading to a simplified and lightweight architecture. ${ }^{[16]}$ In ethanol, these SWCNT films can be easily spread out onto the separator end to end or/and layer by layer. When the SWCNT films were spread out onto the separator end to end, the ends of neighbouring SWCNT films overlap, as shown in Figure 3a. This can effectively avoid the presence of uncovered regions of the separator and ensure good connection between the SWCNT films. Relative to the size of the films, these short overlapping regions have little effect on the uniformity of the SWCNT films on the separator, however, if the overlapping is greater than $1 \mathrm{~mm}$, there is a loss of uniformity in the coating. The quantity and configuration of the SWCNT films on the separator can be adjusted, according to the practical demand. After uniformly spreading out SWCNT films onto the separator, ethanol was evaporated and the SWCNT films adhered firmly to the separator, as shown in Figure 3d. Subsequently, two pieces of separator coated with SWCNT films were stacked together and rolled up (Figure 3e), following which the SC was filled with $1 \mathrm{M} \mathrm{LiClO}_{4}$ in a mixture of ethylene carbonate (EC), diethyl carbonate (DEC), and dimethylene carbonate (DMC) in a volume ratio of $\mathrm{EC} / \mathrm{DEC} / \mathrm{DMC}=1: 1: 1$ as the electrolyte, as shown in Figure $3 \mathrm{f}$. It is worth noting that the shape and size of the external shell of the resulting SC can be readily adjusted to various designs according to the practical applications.

In our case, the SWCNT films were directly used as both electrodes and charge collectors, which not only eliminated the metal/SWCNT film, namely charge collector/electrode interface, but also simplified the SC design with less weight. The SWCNT film-based roll-designed SC was powerful enough to light up a light-emitting diode (Figure $3 \mathrm{~g}$ ) when fully charged. The electrochemical characterization of the above roll-designed SC was investigated by cyclic voltammetry $(\mathrm{CV})$ at different scan rates (Figure 3h), showing a typical rectangular shape. This shape indicates that the SWCNT film has excellent capacitance behaviour with a rapid current response on voltage reversal with 
low equivalent series resistance (ESR), which refers to the resistance that adds to the impedance due to imperfections within the capacitor's material.

The specific capacitance decreases continually with the increase of scan rate due to progressively less efficient infiltration of ions into the porous films at higher scan rates (Figure 3i), a typical characteristic of CNT film electrode due to diffusion limitations. At slower scan rates, the diffusion of ions from the electrolyte can gain access to almost all available pores of the film electrodes and penetration of electrolytic ions into the pores will be greatly deeper, leading to a high specific capacitance. However, when the scan rate is increased, efficient infiltration of ions into the porous films is progressively less due to a decreased time for this diffusion to occur, as a result, there is a reduction in capacitance.

The specific capacitance calculated from charge/discharge curves (Figure 3j) of the compact SWCNT film SC is approximately $35 \mathrm{~F} / \mathrm{g}$ for a two-electrode cell and corresponds to $140 \mathrm{~F} / \mathrm{g}$ for a three-electrode cell, which is higher than those of a post-deposited CNT film or CNT array SCs, the energy density is 43.7 $\mathrm{Wh} / \mathrm{kg}$ and the maximum power density of the resulting compact SWCNT film $\mathrm{SC}$, is $197.3 \mathrm{~kW} / \mathrm{kg}$. The high conductivity of our SWCNT film electrode and the optimized architecture of SCs result in a low ESR and therefore a high power density of the SWCNT film-based SC. In addition, the coulombic efficiency of the compact SC reached $\sim 95 \%$ with a knee frequency of approximately $17 \mathrm{~Hz}$. These results clearly show the potential application of free-standing SWCNT film in compact SCs with enhanced performance and significantly improved energy and power densities.

Although directly grown SWCNT films are freestanding, highly conducting and transparent, the thickness of our freestanding SWCNT films could not be less than $100 \mathrm{~nm}$ otherwise the sample could not be removed, non-destructively, from the wall of the quartz tube. Thus, the transparency of as-prepared SWCNT films could not exceed that of the SWCNT film with a thickness of $100 \mathrm{~nm}$ 
$(70 \%$ at $550 \mathrm{~nm})$ and is lower than that required for many applications, such as touch screens. As such, it is of great significance to reduce the thickness and increase the transparency of as-prepared, directly grown, SWCNT films for broad applications. To decrease the thickness and improve the transparency of directly grown SWCNT films, we report a fast, simple, and effective strategy for post-treatment of directly grown SWCNT films to fabricate ultrathin transparent and conductive films (TCFs) on a flexible and transparent substrate, as shown in Figure 4a. ${ }^{[17]}$ This strategy includes two processes. Firstly, an approach is put forward for processing free-standing SWCNT films to resolve the stickiness problem and spread them out onto various substrates uniformly. Then, a "repeated halving" approach based on electrostatic adsorption is presented to separate a directly grown thick SWCNT film quickly into many ultrathin SWCNT films with a thickness less than $100 \mathrm{~nm}$, and down to a quasi-single layer of SWCNT bundle network. The "repeated halving" approach effectively improves the utilization rate of SWCNTs and can retain the uniformity of SWCNT films. Moreover, this method simplifies experimental procedures and conditions and provides a simple, rapid process to prepare ultrathin SWCNT TCFs with high efficiency and good reproducibility. The thickness of thinnest film can be up to about $13 \mathrm{~nm}$ (Figure 4b). The transmittance of the SWCNT film on the PET substrate obtained by "repeated halving" approach is greatly increased with decreasing film thickness, up to $90 \%$ transmittance (Figure 4c), which is of great significance for the practical applications.

To meet the design and power needs of fully flexible and transparent devices, flexible and transparent energy conversion and storage units or devices have to be considered. The ultrathin SWCNT films prepared by "repeated halving" approach possess high conductivity, transmittance and flexibility, making these suitable for transparent energy conversion and storage devices. As a proof-ofconcept, the ultrathin SWCNT films prepared by "repeated halving" approach were fabricated into flexible and transparent symmetrical SCs (Figure $4 \mathrm{~d}$ and e). 
The separator and electrolyte were sandwiched by the SWCNT films on the PET substrates. Nafion 117 membrane served as transparent separator and the electrolyte is $1 \mathrm{M}$ non-aqueous $\mathrm{LiClO}_{4}$ in a mixture of ethylene carbonate (EC), diethyl carbonate (DEC), and dimethylene carbonate (DMC) in a volume ratio of $E C / D E C / D M C=1: 1: 1$. The transmittance of the SCs using SWCNT films with different thicknesses as electrodes is shown in Figure 4c. The transmittance of SCs fabricated using SWCNT films of approximately $50 \mathrm{~nm}$ in thickness is about $60 \%$ at $550 \mathrm{~nm}$ (Figure $4 \mathrm{f}$ ).

The calculated specific capacitance for the transparent SWCNT electrode material is $22.5 \mathrm{~F} / \mathrm{g}(146 \mu \mathrm{F} / \mathrm{cm}$ for SWCNT films of $50 \mathrm{~nm})$. The energy density of the SWCNT film electrodes is $12.5 \mathrm{Wh} / \mathrm{kg}$ and the maximum power density is $13.9 \mathrm{~kW} / \mathrm{kg}$. In order to study the stability of flexible and transparent SWCNT film SCs, the prepared SCs were examined by galvanostatic charge/discharge measurements for 200 cycles under the same conditions, as shown in Figure 4f. After10 cycles, there is a slight decrease in specific capacitance from 22.5 to $20 \mathrm{~F} / \mathrm{g}$. The specific capacitance then retains the same value with minimal capacitive loss up to 200 cycles.

Based on an organic iron catalyst, iron(III) p-toluenesulfonate (Fe(III)pTS), we modified CVD synthesis to grow CNT networks integrated into a conductive carbon layer (CL) on insulating quartz sheets (Figure 5) ${ }^{[18]}$ This method was further adapted for growth on metallic substrates (e.g. aluminum or copper foil), and on structured carbons such as carbon fibre paper. ${ }^{[19]}$ In a typical experiment, a thin film of $\mathrm{Fe}(\mathrm{III}) \mathrm{pTS}$ catalyst was spin-coated onto quartz plates from organic solutions. After annealing, which facilitates solvent removal, the CVD process was initially carried out at $600{ }^{\circ} \mathrm{C}$ under $\mathrm{Ar} / \mathrm{H}_{2}$ gas flow to reduce the $\mathrm{Fe}^{3+}$ catalyst to iron nanoparticles. Then, a CNT growth phase carried out at $800{ }^{\circ} \mathrm{C}$ using $\mathrm{C}_{2} \mathrm{H}_{2}$ as the carbon source. The resultant CNT films (Figure 5a) were observed to be very much unlike those grown using conventional catalysts. During CNT growth, a reflective layer was formed beneath the carbon film 
(Figure 5b). The formed CNT/carbon layer (CNT/CL) paper appears as a mattblack layer on top of the quartz plate, while a flexible, shiny carbon layer forms the lower layer of the CNT/CL paper. The CNT/CL paper was easily removed from the substrate and the resulting freestanding film could be rolled around a glass rod without visible signs of degradation (Figure 4c). The size of the CNT/CL 3D networks is easily scaled up to $100 \mathrm{~cm}^{2}$ by using a larger CVD quartz tube, given that scale up is not limited by the CVD process itself. SEM confirmed the top layer indeed consisted of CNTs and a highly porous 3D structured CNT network grown on top of a dense CL with less than $1 \mu \mathrm{m}$ thickness (Figure 5d and e). The long nanotubes obtained in the network are MWCNTs with an external diameter of 20-40 nm, and the CL (Figure 5f) displays a uniformly dense continuous film. The resistance of CNT/CL paper is about $46 \Omega$ /square is significantly lower than that of amorphous carbon $(>1$ $\mathrm{k} \Omega$ /square), which is often formed on top of CNTs during normal CVD based growth processes using inorganic iron(III) compounds. These networks possess a high surface area and superior electrochemical properties. The freestanding CNT/CL paper electrodes are lightweight, flexible. Furthermore, they exhibit high conductivity, high surface area and superior electrochemical properties, which make them promising as flexible electrodes.

Activated carbon (AC), which is widely used in commercial products, is a cheap electrode material with a high surface area yielding a moderate capacity for SCs. AC in powder form is difficult to handle as an electrode material. Thus, conductive agents (carbon black) and binder (poly(tetrafluoroethylene) (PTFE)) are always needed. Recently, Dai et al used CNTs to bind AC particles together to obtain a paper-like composite by filtration. ${ }^{[20]}$ The AC/CNT papers possess higher specific capacity, rate performance, and power density than the corresponding values for an AC/acetylene black electrode. The capacity reached a maximum value of $267.6 \mathrm{~F} / \mathrm{g}$ with a CNT loading of $5 \mathrm{wt} \%$, and the energy density and power density were $22.5 \mathrm{Wh} / \mathrm{kg}$ and $7.3 \mathrm{~kW} / \mathrm{kg}$, respectively, at a 
high current density of $20 \mathrm{~A} / \mathrm{g}$. The AC/CNT paper electrode also showed a good cycle performance, with $97.5 \%$ of the original capacity retained after 5000 cycles at a scan rate of $200 \mathrm{mV} / \mathrm{s}$. This method affords not only a promising paper-like nanocomposite for use in low-cost and flexible SCs, but also a general way of fabricating multi-functional paper-like CNT-based nanocomposites for use in devices such as flexible lithium ion batteries and solar cells.

\subsection{Graphene-based flexible EDLCs}

Graphene is a monolayer of planar carbon structured in conjugated hexagonal lattices, in which the carbon bonds are $\mathrm{sp}^{2}$ hybridized. Graphene has attracted increasing attention because of its unique structure and properties. ${ }^{[21-49]}$ For example, in graphene, electron-phonon scattering is so weak that the mobilities of charge carriers can be improved significantly, even up to $200000 \mathrm{~cm}^{2} / \mathrm{Vs}$ under ambient conditions, if the extrinsic disorder is eliminated. ${ }^{[50]}$ This value exceeds the intrinsic mobility of any other semiconductor. ${ }^{[50-51]}$ In addition, graphene possess a large theoretical specific surface area $\left(2630 \mathrm{~m}^{2} / \mathrm{g}\right),{ }^{[52]} \mathrm{high}$ Young's modulus $(\sim 1.0 \mathrm{TPa}),{ }^{[53]}$ thermal conductivity $(\sim 5000 \mathrm{Wm} / \mathrm{K})^{[54]}$, and good electrical conductivity. ${ }^{[55]}$ Its unique structure and properties make graphene promising for a EDLC electrodes. ${ }^{[56]}$ Although pure graphene sheets with high quality can be prepared by mechanical cleavage of graphite ${ }^{[57]}$ or CVD method at high temperature, ${ }^{[58-76]}$ these methods suffer from high cost, high temperature, substrate limitation, and extremely low yield. In addition, the surface of pristine graphene is so hydrophobic that pristine graphene tend to agglomerate in solvents, leading to the loss of excellent properties of monolayer graphene sheets. As a result, solution-based process in a large scale is not applicable for the graphene sheets obtained from above mentioned methods. Solution-phase exfoliation of graphite is an effective method for the mass production of graphene and its derivatives. ${ }^{[77-81]}$ Graphene derivatives such as graphene oxide (GO), an electrical insulator, and reduced graphene oxide (rGO), 
lose planarity and electrical conductivity through functionalization disrupting the conjugation of the basal plane. However, these materials become significantly easier to process into usable materials as a trade-off. ${ }^{[22]}$

Ruoff and co-workers first explored graphene-based EDLCs using rGO as electrode materials. ${ }^{[52]}$ Although these rGO-based electrodes exhibit excellent performance, these graphene-based electrodes have been achieved by mixing rGO with conductive binders and coating collector electrodes. The utilization of conductive binders and metal substrates would limit the flexibility of the SC electrodes. Therefore, the integration of graphene sheets, into flexible paper-like structures (e.g., layered films and porous scaffolds) is drawing much attention. However, in most cases, during the assembly process of nanoscale building blocks into paper-like structures, the large accessible surface area of 2D graphene sheets is lost. The reason for this loss is that the individual graphene sheets irreversibly aggregate and restack due to the strong $\pi$ - $\pi$ stacking and van der Waals force between the planar basal planes of graphene sheets. This affects the potential applications of graphene materials in electrochemical electrodes, composite materials, and so on. Therefore, preventing aggregation of graphene sheets in macroscopic porous structures, such that the properties of the individual graphene sheets are not compromised, is a critical challenge in constructing functional graphene-based macroscopic structures for SC electrodes.

Leavening is the process of adding gas to produce lighter, more easily chewable bread with the porous structures from compact dough before or during baking or steaming. Inspired from this, we fabricated paper-like, lightweight, and electrically conductive rGO foams with open porous and continuous crosslink structures by autoclaved leavening and steaming of GO layered films, as depicted in Figure 6a and $6 b^{[83]}$ We clearly observed the formation of foamed structures with $\sim 50$ times the volume expansion and $\sim 30 \%$ of the mass loss in comparison with original GO film. The porous walls in the foams are 
continuously cross-linked and not simply and completely separated between different layers, as shown in Figure 6c. Such continuous cross-linked structures not only effectively overcome the re-stacking forces between graphene sheets, but also allow for better conductive contacts between the sheets to lower the resistance of foams (to less than $100 \Omega$ /square). Furthermore, the porous walls consisting of thin layers of stacked graphene sheets in these foams are not fully compact and there are still small gaps in the walls, allowing for electrolyte diffusion through the materials. The tensile strength of the rGO foams is about 3.2 $\mathrm{MPa}$ and the Young's modulus of the rGO foams were about 7 and $40 \mathrm{MPa}$ at the first and last tensile stage, respectively. The tensile strain can be up to $10 \%$, which is much larger than the case of the compact GO films $(<0.6 \%)$. Since the free-standing $\mathrm{rGO}$ foams with continuous cross-linking porous structures overcome the re-stacking forces, it results in higher active electrochemical surface area and good conductivity. Our rGO foams possess a porous structure coupled with high mechanical strength and conductivity. These properties make our rGO foams an excellent candidate for flexible SC electrodes where neither an insulating binder nor a low capacitance conducting additive is required. In a proof-of-concept experiment, we built flexible rGO foam SCs using free-standing rGO foams as both current collectors and electrodes. The free-standing rGO foams were first uniformly spread out onto a PET substrate. Then, the separator and electrolyte were sandwiched by the rGO foams on the PET substrates, as shown in Figure $6 \mathrm{~d}$ and e. Here, filter paper served as separator and the electrolyte was $1 \mathrm{M} \mathrm{H}_{2} \mathrm{SO}_{4}$. CVs of the $\mathrm{SC}$ were found to be rectangular in shape within a selected range of potential (Figure 6f), indicating an excellent capacitive behavior. The discharge curve is nearly linear (Figure $6 \mathrm{~g}$ ), indicating good electrical double layer performance. The calculated specific capacitance of the resulting rGO foam SC is about $110 \mathrm{~F} / \mathrm{g}$. The calculated specific capacitance of SC based on rGO foams is much larger than that of SCs using compact $\mathrm{rGO}$ films as electrodes $(17 \mathrm{~F} / \mathrm{g})$. The knee frequency of rGO 
foam electrodes is $15 \mathrm{~Hz}$ (Figure $6 \mathrm{~h}$ ). $\mathrm{CV}$ curves of a representative rGO foam SC (device size: $3 \mathrm{~cm} \times 3 \mathrm{~cm}$, rGO foam electrode size: $1.5 \mathrm{~cm} \times 1.5 \mathrm{~cm}$ ) before and after bending demonstrated nearly no deviation (Figure 4f) when the distance between two sides of the $\mathrm{SC}$ was changed from 3 to $2 \mathrm{~cm}$ under bending (Figure 6i). This suggested that the rGO foam SCs are quite stable under bending, and the rGO foams are suitable for the flexible device applications.

Li et al successfully fabricated stable aqueous rGO sheet colloids through electrostatic stabilization. ${ }^{[82]}$ Conductive rGO films can also be readily formed by vacuum filtration of the above rGO dispersions and subsequent drying procedures. However, if the rGO samples were not fully dried, the graphene sheets can remain largely separated in a solvated state, providing a highly open pore structure (Figure 7a), allowing the electrolyte solution to easily access to the surface of individual sheets. This has made it possible to combine ultrahigh power density and high energy density in graphene-based SCs and to allow the device operational at high rates. ${ }^{[84]}$ In an aqueous electrolyte, specific capacitance of rGO film is up to $215.0 \mathrm{~F} / \mathrm{g}$ (Figure $7 \mathrm{~b}$ ). A capacitance of 156.5 $\mathrm{F} / \mathrm{g}$ can be obtained even at an ultrafast charge/discharge rate of $1080 \mathrm{~A} / \mathrm{g}$. These RGO film can provide a maximum power density of $414.0 \mathrm{~kW} / \mathrm{kg}$ at a discharge current of $108 \mathrm{~A} / \mathrm{g}$. The operating frequency of the rGO film is $75 \mathrm{~Hz}$. In an ionic liquid electrolyte, rGO film-based SCs can offer a specific capacitance of up to $273.1 \mathrm{~F} / \mathrm{g}$ and an energy density and maximum power density up to $150.9 \mathrm{Wh} / \mathrm{kg}$ and $776.8 \mathrm{~kW} / \mathrm{kg}$, respectively (Figure $7 \mathrm{~b}$ ). RGO films can be bonded on to shape-memory polyurethane (PU) film and forming a bi-layered composite film. ${ }^{[85]}$ Based on the good flexibility of graphene paper and the outstanding shape holding capacity of PU phase, the resulting composite film can be changed into various shapes. The rGO/PU composite film used as the supercapacitor electrode maintains a satisfactory electrochemical capacitance. Su et al prepared a 3D macroporous bubble rGO film through a 
novel hard template-directed ordered assembly by combining vacuum filtration and polymethyl methacrylate (PMMA) latex spheres. ${ }^{[86]}$ The as-prepared rGO film provides mediated 3D macropores for ion-buffering reservoirs and lowresistant channels for ion diffusion, which are promising characteristics for a high rate supercapacitor electrode. The supercapacitors based on 3D macroporous bubble rGO films exhibit extraordinary electrochemical capacitance with high rate capability.

Laser Irradiation of the GO film induces the reduction of GO into rGO with improving electrical conductivity. Ajayan et al demonstrated the ability to directly pattern various $\mathrm{rGO}$ geometries with micrometre resolution on a single piece of GO paper for the construction of both in-plane and conventional sandwich SC designs as shown in Figure 7d-g. ${ }^{[87]}$ The substantial amounts of trapped water in the GO makes GO simultaneously a good ionic conductor and an electrical insulator, serving as both an electrolyte and an electrode separator with ion transport characteristics. The in-plane SC structure (Figure 7d) with a circular geometry gives the highest capacitance of $0.51 \mathrm{mF} / \mathrm{cm}^{2}$, nearly twice that of the sandwich structure (Figure 7e). The energy density for in-plane SC device is calculated to be $\sim 4.3 \times 10^{-4} \mathrm{Wh} / \mathrm{cm}^{3}$, with a power density of $1.7 \mathrm{~W} / \mathrm{cm}^{3}$ (Figure 7f). The sandwich device geometry shows a higher power density of $\sim 9.4 \mathrm{~W} / \mathrm{cm}^{3}$ due to its lower ESR value, however, the energy density is lower $\left(1.9 \times 1024 \mathrm{Wh} / \mathrm{cm}^{3}\right)$. The cyclic stability tests shows that, after 10,000 cycles, there is a $\sim 30 \%$ drop in the capacitance of the in-plane circular device, whereas an $\sim 35 \%$ drop for the sandwich devices. Based on a standard LightScribe DVD optical drive to do the direct laser reduction of graphite oxide films to graphene, as depicted in Figure $7 \mathrm{~h},{ }^{[88]}$ Kaner and co-workers prepared a rGO film with an open network. The as-prepared films with open pores are mechanically robust, show high electrical conductivity $(1738 \mathrm{~S} / \mathrm{m})$ and specific surface area (1520 $\mathrm{m}^{3} / \mathrm{g}$ ), indicating that the films can be used directly as EC electrodes without the need for binders or current collectors. The SCs based on laser-scribed rGO films 
are robust enough to be charged and discharged over a wide range of scan rates (from 100 to $10,000 \mathrm{mV} / \mathrm{s}$ ) and still maintain its nearly ideal rectangular $\mathrm{CV}$ shape. The area capacitance of the laser-scribed $\mathrm{rGO}$ SC was $3.67 \mathrm{mF} / \mathrm{cm}^{2}$ in polymer electrolyte and $4.04 \mathrm{mF} / \mathrm{cm}^{2}$ in $1.0 \mathrm{M} \mathrm{H}_{2} \mathrm{SO}_{4}$ at $1 \mathrm{~A} / \mathrm{g}$ (Figure $7 \mathrm{i}$ ). The area capacitance of the $\mathrm{SC}$ device is maintained at $1.84 \mathrm{mF} / \mathrm{cm}^{2}$, even when the $\mathrm{SC}$ is operated at an ultrafast charge/discharge rate of $1000 \mathrm{~A} / \mathrm{g}$, indicating a very high rate capability. The laser-scribed rGO SC exhibits energy densities of up to $1.36 \mathrm{mWh} / \mathrm{cm}^{3}$ and power density of $\sim 20 \mathrm{~W} / \mathrm{cm}^{3}$.

Ruoff et al present a novel method to prepare highly conductive, freestanding, and flexible porous carbon thin films by chemical activation of rGO paper by $\mathrm{KOH} .{ }^{[89]}$ First, $1 \mathrm{M} \mathrm{KOH}$ was dropwise added into $1 \mathrm{mg} / \mathrm{mL}$ of GO colloidal suspension. The water was evaporated by heating in an oil bath at 100 ${ }^{\circ} \mathrm{C}$ under constant stirring until it thickened into an "ink paste". The paste consists of $\mathrm{GO}$ platelets dispersed with $\mathrm{KOH}$ in some remaining water. Films composed of stacked and overlapped GO platelets decorated with $\mathrm{KOH}$ were obtained through brief vacuum filtration under directional flow followed by drying, followed by an activation step, in which the dry film was placed in a quartz tube furnace under flowing argon at $800{ }^{\circ} \mathrm{C}$ for $1 \mathrm{~h}$ and a pressure of 1 atm. These flexible carbon thin films possess a very high specific surface area of $2400 \mathrm{~m}^{2} / \mathrm{g}$ with a high in-plane electrical conductivity of $5880 \mathrm{~S} / \mathrm{m}$. These rGO films demonstrated an excellent high-frequency response, an extremely low ESR, on the order of $0.1 \mathrm{Ohm}$, and a high-power delivery of about $500 \mathrm{~kW} / \mathrm{kg}$. The specific capacitances and energy densities of these rGO thin films are 120 $\mathrm{F} / \mathrm{g}$ and $26 \mathrm{~W} / \mathrm{h} \mathrm{kg}$, respectively. Free-standing thin films provide a route to simplify the electrode-manufacturing process by eliminating conducting additives and binders and are also compatible with roll-to-roll thin-film fabrication technologies.

A multilayered nano-architecture of variable sized graphene nanosheets was prepared by capillary force driven LBL self-assembly. ${ }^{[90]}$ A two-phase mixture 
was first formed by dispersing Graphene nanosheets in chloroform followed by the addition of a small amount of water. This two-phase mixture was then briefly sonicated to get the nanosheets adsorbed at the liquid-liquid interface. Once the nanosheets were adsorbed at the interface, they could not be brought back to the bulk phase even after vigorous shaking. However, upon shaking, numerous emulsion droplets with the nanosheets were formed still covering the interface between the two immiscible liquids. After these droplets reach the airwater interface, they spread in the form of a thin film. Following by the rapid evaporation of chloroform, a dry graphene film was obtained and floated at the air-water interface. This film can be easily transferred to other substrate. To remove the residual water, the film was then heated at $100{ }^{\circ} \mathrm{C}$. Multilayer films were formed by successive depositions of these monolayer films on top of another. The multilayer graphene film exhibits very high electrical conductivity of $1.24 \times 10^{4} \mathrm{~S} / \mathrm{m}$. This multilayer graphene film exhibits a high-frequency capacitative response and a nearly rectangular cyclic voltammogram at a 1000 $\mathrm{mV} / \mathrm{s}$ scan rate and possesses a rapid current response. The average specific capacitance of the aligned composite configuration was close to $80 \mathrm{~F} / \mathrm{g}$ at a high charge/discharge current $10 \mathrm{~A} / \mathrm{g}$. The SCs based on this multilayered film structure demonstrate long term stability with the retention of more than $98 \%$ of the specific capacitance at the end of 1000 electrochemical cycles. In addition, the resulting multilayer film exhibits superior high-frequency capacitative properties with a knee frequency close to $398 \mathrm{~Hz}$.

To overcome the self-agglomeration of graphene sheets in macroscale structures, we report a facile and scalable assembly strategy, the electrophoretic alternate deposition, to fabricate alternately multilayered films using graphene sheets and metal nanoparticles as building blocks, as depicted in Figure 8a and b. ${ }^{[91]}$ The conductivity of hybrid films can be up to $8375 \mathrm{~S} / \mathrm{m}$, which is larger than other porous $\mathrm{rGO}$ structure $(0.5 \mathrm{~S} / \mathrm{m})$. In the multilayered $\mathrm{rGO} / \mathrm{AuNP}$ hybrid nano-architecture, AuNP layers not only avoid the self-agglomeration of 
rGO sheets, but also connected the rGO layers well. This assembly strategy can effectively control the orientation of graphene nanosheets and metal NPs and the organization at the nanoscale. The obtained multilayered films on ITO substrates can easily be transferred to other flexible substrates, which is a fundamental requirement for flexible devices

Since these $\mathrm{rGO} / \mathrm{AuNP}$ hybrid films have a higher electrical conductivity, the hybrid films are expected to be used as the electrodes of flexible SC devices. Here, neither an insulating binder nor a low capacitance conducting additive is required. We constructed and measured the performance of two-electrode symmetrical flexible SC cells based on the obtained multilayered $\mathrm{rGO} / \mathrm{AuNP}$ hybrid films. The separator (Celgard 2325) and electrolyte were sandwiched by the rGO/AuNP films on the PET substrates (Figure 8c and d). To make SCs more flexible, directly grown SWCNT films were used as current collectors instead of metallic foils or foams, as directly fabricated SWCNT films have high conductivity, low mass density, large specific surface area and high mechanical strength. In traditional SC devices, metallic foils or foams are normally used as current collectors for both anode and cathode due to poor conductivity of active electrode materials. However, the use of metallic current collectors would make the SCs too heavy, bulky or inflexible, which limit their use in applications where space and weight are constraints. The mass density of SWCNT film is much smaller than that of metallic current collectors, leading to a lightweight architecture. In addition, the porosity of SWCNT films may be useful to improve the penetration of electrolytic ions into the electrodes, leading to a high specific capacitance. The calculated specific capacitance, energy and maximum power densities of the resulting multilayered hybrid film from CV (Figure 8c) and discharge curves (Figure 8 d) SC were $65 \mathrm{~F} / \mathrm{g}, 36 \mathrm{Wh} / \mathrm{kg}$ and $49 \mathrm{~kW} / \mathrm{kg}$, respectively, which is higher than that of SCs based on compact RGO films (23 $\mathrm{F} / \mathrm{g}, 13 \mathrm{Wh} / \mathrm{kg}$ and $27 \mathrm{~kW} / \mathrm{kg}$ ). These indicate that the addition of the spacer, AuNPs, in the multilayered film effectively improve the performance of the rGO 
$\mathrm{SC}$ electrodes. Furthermore, it is important to note that the specific capacitances of SCs based on the RGO/AuNP hybrid films is strongly related to the AuNP deposition time, as shown in Figure 8g. The optimal deposition time for AuNPs was determined to be 120 s with deposition times on either side of this value resulting in a decreased capacitive response.The RGO/AuNP hybrid film SCs possess high knee frequency of $370 \mathrm{~Hz}$, as show in Figure 8h. It was found that there was nearly no obvious deviation in the CV curves (Figure 8i) when the distance between two sides of the supercapacitor was changed from 3 to $2 \mathrm{~cm}$ under bending. This suggested that the hybrid film supercapacitors are quite stable under bending, and the hybrid film are suitable for the flexible device applications.

Apart from Au NPs, carbon black (CB) NPs can also be used as "spacer" to prevent the restacking of graphene sheets in the graphene film. ${ }^{[92]}$ Flexible graphene paper (GP) pillared by CB NPs was produced using a simple vacuum filtration method. The pillared GP can be directly used as SC electrodes without binder, serving both as electrodes and current collectors, due to its intrinsic high electrical conductivity. The pillared GP-based SCs exhibit excellent electrochemical performances and cyclic stabilities, compared to GP without the addition of CB nanoparticles. The specific capacitance of the pillared GP is 138 $\mathrm{F} / \mathrm{g}$ and $83.2 \mathrm{~F} / \mathrm{g}^{-}$in aqueous and organic electrolytes, respectively, at a scan rate of $10 \mathrm{mV} / \mathrm{s}$. After 2000 cycles, a negligible amount of capacitance loss was observed in aqueous and organic electrolytes, $3.85 \%$ and $4.35 \%$, respectively. At an extremely fast scan rate of $500 \mathrm{mV} / \mathrm{s}$ the specific capacitance can reach 80 $\mathrm{F} / \mathrm{g}$ in aqueous electrolyte. The pillared GP has great potential in the development of promising flexible and ultralight-weight SCs for electrochemical energy storage.

To prepare flexible and transparent graphene EDLCs, Chen and co-workers fabricated flexible and transparent graphene films via vacuum filtering. ${ }^{[93]}$ The minimum thickness of the produced graphene films was up to $25 \mathrm{~nm}$. At $550 \mathrm{~nm}$ 
the highest transmittance of approximately $70 \%$ was obtained for a $25 \mathrm{~nm}$ thick film. The conductivities of the films range from 800 to1000 S/m. The film collected on a PET substrate can be readily bent to some degree without losing conductivity. The transparency, conductivity, and flexibility of graphene ultrathin films demonstrate it as a promising material for application where flexible coatings are required, such as flexible electrodes. At a scan rate of 10 $\mathrm{mV} / \mathrm{s}$, specific capacitances of $111 \mathrm{~F} / \mathrm{g}, 105 \mathrm{~F} / \mathrm{g}, 102 \mathrm{~F} / \mathrm{g}$, and $99 \mathrm{~F} / \mathrm{g}$ were obtained for the $25 \mathrm{~nm}, 50 \mathrm{~nm}, 75 \mathrm{~nm}$, and $100 \mathrm{~nm}$ films, respectively. There is a slight increase of $12 \mathrm{~F} / \mathrm{g}$ in specific capacitance as the film thickness decreases from 100 to $10 \mathrm{~nm}$. It is important to note that the graphene thin films retain $75 \%-80 \%$ of their capacitance when the scan rate is increased from 10 to 200 $\mathrm{mV} / \mathrm{s}$. This suggests that there is very good contact between the electrolyte and the active material allowing for excellent charge transfer kinetics. The $25 \mathrm{~nm}$ film shows the best performance with a high energy density of $15.4 \mathrm{Wh} / \mathrm{kg}$ at $554 \mathrm{~W} / \mathrm{kg}$ which gradually reduces to $10 \mathrm{Wh} / \mathrm{kg}$ at $7200 \mathrm{~W} / \mathrm{kg}$.

Cotton cloth has been used to support SWCNT as flexible SC electrodes due to the flexible and porous structure made by weaving natural cotton fibers, which leads to a hierarchical network structure with a complicated surface morphology, functional groups such as hydroxyl groups, and high porosity. Yan et al prepared a flexible, processable, electrode via a simple " brush-coating and drying" process using everyday cotton cloth as the platform and a stable GO suspension as the ink. ${ }^{[94]}$ Following by annealing at $300{ }^{\circ} \mathrm{C}$ in argon atmosphere, the graphene/cotton cloth composite fabric exhibits good electrical conductivity, outstanding flexibility, and strong adhesion between graphene nanosheets (GNSs) and cotton fibers. The SC based on graphene/cotton cloth composite fabric shows a specific capacitance of $81.7 \mathrm{~F} / \mathrm{g}$ for a two-electrode system in aqueous electrolyte. In an ionic-liquid/organic electrolyte, the SC also exhibits satisfactory capacitance of $83.2 \mathrm{~F} / \mathrm{g}$. Using pure cotton cloth as separator and GNSs/cotton cloth composite fabric as electrode and current collector, an all- 
fabric SC was also fabricated. Such a conductive GNSs/cotton cloth composite fabric may provide new design opportunities for wearable electronics and energy storage applications. In addition, a flexible and freestanding carbon nanofiber/graphene nanosheet (CNF/GNS) composite paper was prepared via electrospinning followed by high-temperature annealing using a polyacrylonitrile/GNS/dimethylformamide mixture as electrospun precursor. ${ }^{\text {[95] }}$ The GNS/CNF composite paper exhibits the largest specific capacitance of 197 F/g, about $24 \%$ higher than that of pure CNF paper. Therefore, based on the above investigations, such GNS/CNF composite paper can be a potential candidate for high-performance flexible capacitors.

The introduction of an electric phase into a cellulose paper can make it electrically active for applications such as electrodes, electromagnetic shielding and sensors. High performance flexible cellulose electric papers can be used for the development of novel paper electronics and foldable energy storage devices. GNS coated cellulose composite papers can be fabricated via a simple papermaking process, dispersing chemically synthesized GNS into a cellulose pulp, followed by filtration. ${ }^{[96]}$ These composite papers are electrically conductive and electrochemically active. The GNS/cellulose paper is as flexible and mechanically tough as the pure cellulose paper. The electrical measurements show the composite paper has a sheet resistance of $1063 \Omega /$ square and a conductivity of $11.6 \mathrm{~S} / \mathrm{m}$. As a flexible double layer SC electrode, the GNS/cellulose paper in an organic electrolyte $\left(\mathrm{LiPF}_{6}\right)$ displays a high capacity of $252 \mathrm{~F} / \mathrm{g}$ at a current density of $1 \mathrm{~A} / \mathrm{g}$ with respect to GNS.

\subsection{CNT-Graphene Composites}

As mentioned above two sections, pure rGO or CNTs can be directly used as SC electrodes. The SC electrodes based on CNTs possess high power density due to high conductivity of CNTs. The SC electrodes based on $\mathrm{rGO}$ sheets have high specific capacitance due to the high specific surface area of rGO sheets. 
The combination of CNTs and rGO sheets is a compromise between the high conductivity of CNTs and the high specific surface area of RGO. A transparent, conductive, and flexible MWCNT/rGO hybrid film with an interconnected network and a double-layer structure can be prepared by by four steps: 1) vacuum-filtration of an aqueous MWCNT and GO solution onto a membrane; 2) transferring onto a PET sheet by dissolving the membrane in acetone; 3) chemically reducing graphene oxide to graphene by $\mathrm{HI}$ and repeatedly washing by ethanol; 4) $\mathrm{HNO}_{3}$ treatment for the p-type doping and the removal of the surfactant. ${ }^{[97]}$ The thickness and transparency can be tuned by varying the $\mathrm{CNT} / \mathrm{rGO}$ ratios and the volumes of the solution. Furthermore, this MWCNT/rGO hybrid flexible film maintains an excellent stability of the conductivity (over 95\%) after the repeated flexure over $120^{\circ}$ more than 3000 times. This stability and performance of the MWCNT/rGO hybrid flexible films indicates a broad applicability for flexible optoelectronics. Interconnected MWCNT/rGO composites with a large surface area and high porosity show a maximum specific electrochemical capacitance of $150 \mathrm{~F} / \mathrm{g}$, which is much higher than that for only MWCNT $(100 \mathrm{~F} / \mathrm{g})$. Importantly, the MWCNT/graphene exhibits a high rate capability of $141 \mathrm{~F} / \mathrm{g}$ at high current density $(10 \mathrm{~A} / \mathrm{g})$ with $96 \%$ retention. The outstanding performance of the MWCNT/rGO hybrid films results from a unique interconnected nanostructure with the highly porous flakes leading to a large surface area. This combination of nanostructure and surface area demonstrates a synergic effect that improves the penetration of electrolyte ions, and the ion transport within a short distance.

Similarly, flexible rGO/MWCNT films can be fabricated by flow-directed assembly from dispersions of GO and pristine MWCNTs followed by the use of gas-based hydrazine or heat-treatment to reduce the GO into RGO.$^{[98-99]}$ The MWCNTs in the hybrid films not only efficiently increase the basal spacing but also bridge the defects for electron transfer between RGO sheets, increasing electrolyte/electrode contact area and facilitating transportation of electrolyte ion 
and electrons to the inner region of electrodes. The rGO/MWCNT films possesses a specific capacitance of $265 \mathrm{~F} / \mathrm{g}$ at $0.1 \mathrm{~A} / \mathrm{g}$ and a good rate capability (49\% capacity retention at $50 \mathrm{~A} / \mathrm{g}$ ), and displays an excellent specific capacitance retention of $97 \%$ after 2000 continuous charge/discharge cycles. ${ }^{\text {[98] }}$

CNTs can form stable aqueous through charge stabilization after acid oxidation, leading to a negative charge on the backbone of the CNT. Stable aqueous dispersions of positively charged polymer-modified graphene sheets were obtained via the in situ reduction of exfoliated graphite oxides in the presence of cationic poly(ethyleneimine) $(\mathrm{PEI}) .{ }^{[100]}$ The resulting water-soluble PEI-modified graphene sheets were then used for sequential self-assembly with acid-oxidized MWCNTs, forming multilayered RGO/CNT hybrid films with interconnected carbon structures of well-defined nanoscale pores. The hybrid films exhibited a nearly rectangular cyclic voltammogram even at a high scan rate of $1 \mathrm{~V} / \mathrm{s}$ with an average specific capacitance of $120 \mathrm{~F} / \mathrm{g}$.

\section{Carbon/Polymer Composite Flexible Pseudo-capacitors}

According to the storage mechanism, pure CNTs- and/or rGO-based SCs are ascribed to EDLCs, which depend on their SSA. However, the major problem of pure CNTs- and/or rGO-based electrodes is that not all their BET surface area is electrochemically accessible when in contact with electrolyte. ${ }^{[5]}$ As a result, their energy density is relatively low. To improve their energy density, the pseudo-capacitance from CP, such as PANI or PPy, and MOs is often coupled with the EDLC to enhance the overall capacitance of the electrode materials. The doped CNTs and/or rGO electrodes display high energy density due to pseudo-capacitance originating from the $\mathrm{CP}$ or MO.

\subsection{CNTs/conductive polymer flexible Pseudo-capacitors}

In section 2.1, it has been shown that pure SWCNT film SC electrodes are usually regarded as the material of choice for a high-power electrode because of their good electrical conductivity and readily accessible surface area. ${ }^{[6,16,101]}$ 
However, the specific surface area of the pure SWCNT film-based electrodes is generally low because SWCNTs exist in the form of bundles, leading to a low energy density. ${ }^{[1,102]}$ Currently, many efforts have been made to improve the energy density of the SWCNT-based electrodes including the addition of CPs into the SWCNT-based electrodes. ${ }^{[103-114]}$ The SWCNT/CP electrodes display high energy density due to pseudo-capacitance originating from CP. Therefore, one of the most critical aspects in the development of SWCNT/CP SCs is to optimize the energy density without deteriorating their high power capability as both these parameters determine the ultimate performance of the SC.

PANI has been considered as one of the most promising electrode materials because of its relatively high conductivity and lower cost compared to other

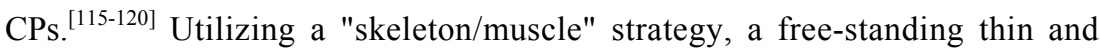
flexible SWCNT/PANI hybrid film was produced via a simple in situ electrochemical polymerization method. ${ }^{[121]}$ The films with a continuous reticulate structure were utilized as a template, as depicted in Figure 9a. ${ }^{[121]}$ In situ electrochemical polymerization effectively deposited PANI onto the surface of SWCNT bundles, and controlled the morphology and microstructure of the SWCNT/PANI hybrid film. In a SWCNT/PANI hybrid film, the directly grown SWCNT film with its continuous reticulate architecture acts as the skeleton and PANI layers act as muscles. This unique continuous "skeleton/muscle" structure (Figure 9b and c) ensures that these hybrid films have much higher conductivity compared to other methods of producing SWCNT/PANI composite films. Furthermore, the continuous "skeleton/muscle" architecture ensures that the SWCNT/PANI hybrid films possess high conductivity. The thin SWCNT/PANI hybrid films remain freestanding and can be bent, rolled up and even twisted without cracking (Figure 9d). As a promising carbon material for flexible SCs, the as-prepared thin SWCNT/PANI hybrid films combining the high conductivity from SWCNT film and the pseudo-capacitance from PANI are suitable for the electrodes of 
flexible SCs in which neither an insulating binder nor a low capacitance conducting additive is required. The flexible SCs have been fabricated directly using the SWCNT/PANI hybrid films as both electrodes and charge collectors without metallic current collectors (Figure 9e). The CV (Figure 9f) curve area of the SWCNT/PANI hybrid film is much larger than that of the pure SWCNT film, which indicates that the specific capacitance of the SWCNT/PANI hybrid film is much higher than that of the pure SWCNT film owing to its additional pseudo-capacitance. CVs of the SC maintain its shape within a selected range of potential and even at a high scan rate of 500 $\mathrm{mV} / \mathrm{s}$ (Figure 9g), indicating that the SWCNT/PANI hybrid films have very rapid current response on voltage reversal. The discharge curves were not ideal straight line (Figure 9h), indicating the process of a faradic reaction. The specific capacitance of SWCNT/PANI hybrid film with 30s of PANI deposition time is $236 \mathrm{~F} / \mathrm{g}$, which is much larger than that of pure SWCNT film $(23.5 \mathrm{~F} / \mathrm{g})$ (Figure 9i), indicating that the specific capacitance of SWCNT film was remarkably improved due to the pseudo-capacitance contributed by PANI. High energy and power densities ( $131 \mathrm{Wh} / \mathrm{kg}$ and $62.5 \mathrm{~kW} / \mathrm{kg}$, respectively) have been achieved. The knee frequency of SWCNT/PANI hybrid film SCs is $25 \mathrm{~Hz}$ (Figure 9j), indicating that most of its stored energy is accessible at frequencies below $25 \mathrm{~Hz}$ for the SWCNT/PANI hybrid film SCs. The high electrical conductivity and flexibility, in combination with a continuous porous architecture, suggest that the as-prepared ultrathin free-standing SWCNT/PANI hybrid films have the significant potential to be the promising electrode materials for thin, lightweight and flexible energy storage devices with high performance.

Freestanding CNT Bucky paper can also be prepared by vacuum filtering. Based on the CNT Bucky paper, Liu et al fabricated the free-standing CNT/PANI hybrid films by chemical polymerization. ${ }^{[105]}$ Compared with the conventional brittle CNT/PANI composites, these paper-like composites were 
significantly thinner and more flexible. The paper-like CNT/PANI film can be rolled up, bent or twisted easily, and even folded without any cracking. These film electrodes showed superior electrochemical performance with a higher specific capacitance $(424 \mathrm{~F} / \mathrm{g})$, lower internal resistivity, and greater stability under different current loads. To simplify the structure and improve the flexibility of SCs, this group developed a ultrathin all-solid-state SC configuration through an extremely simple process based on two slightly separated CNT/PANI electrodes embedded in the $\mathrm{H}_{2} \mathrm{SO}_{4}$-polyvinyl alcohol (PVA) gel electrolyte. ${ }^{[105]}$ The integrated device shows a high specific capacitance of $350 \mathrm{~F} / \mathrm{g}$ for the electrode materials, excellent cycle stability after 1000 cycles and a leakage current as small as $17.2 \mu \mathrm{A}$. Furthermore, due to its polymer-based component structure, it has a specific capacitance of as high as $31.4 \mathrm{~F} / \mathrm{g}$ for the entire device, which is more than 6 times that of current highlevel commercial SC products.

As mentioned above, flexible electrodes have been successfully prepared by uniformly coating SWCNT ink on cotton and other fabrics. However, their specific capacitance is relatively low. To improve the energy density of SWCNT/cloth electrodes, conducting PANI nanowire arrays are successfully deposited onto the surface of the SWCNT/cloth composite through dilute polymerization to obtain the PANI/SWCNT/cloth composite electrodes. ${ }^{[122]}$ The sheet resistance of the PANI/SWCNT/cloth electrode is $150 \Omega /$ square. Using $\mathrm{PANI} / \mathrm{SWCNT} / \mathrm{cloth}$ composites as electrodes, flexible SCs were produced in a sandwich configuration. These flexible composites can be directly used as SC electrodes without using any insulator binder; which simplifies the assembly in practical applications. Bending the flexible SCs to an angle smaller than $90^{\circ}$ did not noticeably affect their performance. The PANI/SWCNT/cloth electrodes display a large capacitance $(410 \mathrm{~F} / \mathrm{g})$, high rate capability, and good stability, which may lead to its future application in high-performance flexible devices. 
To simplify the assembly, flexible sheets of SCs were prepared based on painting a sheet of flexible plastic electrolyte with a composite material made of a CP and CNTs. ${ }^{[123]}$ PVA and phosphoric acid $\left(\mathrm{H}_{3} \mathrm{PO}_{4}, 85 \%\right)$ films, $0.3 \mathrm{~mm}$ thick, were formed by first casting a mixture of PVA and $\mathrm{H}_{3} \mathrm{PO}_{4}$ on a glass surface and then peeling film off the substrate once dry. A painting brush was used to transfer the PANI-CNT aqueous mixture on both sides of a PVA film to form the shape of the SC. Painted sheets were put in an oven at $40{ }^{\circ} \mathrm{C}$ to remove residual water. The specific capacitance of the flexible sheets was evaluated to be $16 \mathrm{~F} / \mathrm{g}$, consisting of pseudo-capacitance produced by the polymer and electrical double-layer capacitance produced by CNTs. The SC sheet has a maximum specific energy of $0.5 \mathrm{Wh} / \mathrm{kg}$ and a maximum specific power of 0.3 $\mathrm{kW} / \mathrm{kg}$. These values are much lower than those observed previously with other forms of flexible SCs. ${ }^{[6,13]}$

To improve the energy density of transparent CNT film SCs, Chen et al presents transparent and flexible SCs assembled from PANI/SWCNT hybrid films, which were prepared by electrochemical polymerization of PANI onto thin SWCNT films. ${ }^{[109]}$ The PANI/SWCNT hybrid films are optically homogeneous, transparent, electrically conducting and mechanically flexible. Owing to the double-layer capacitance from SWCNTs and pseudo-capacitance from PANI, The PANI/SWNT hybrid films show a large specific capacitance of $55.0 \mathrm{~F} / \mathrm{g}$ at a current density of $2.6 \mathrm{~A} / \mathrm{g}$, indicating its possibility for transparent and flexible energy storage.

Apart from PANI, we prepared free-standing poly (3,4ethylenedioxythiophene) (PEDOT) /poly(styrene sulfonate) (PSS)/SWCNT hybrid films with varying compositions of PEDOT/PSS. ${ }^{[124]}$ A comparison of results shows that having single walled nanotubes dispersed throughout the polymer matrix increase the capacitance by $65 \%$ and the energy density by a factor of 3 whilst achieving good capacity retention over 1000 cycles. 


\subsection{Graphene/conductive polymers for flexible pseudo-supercapacitors}

Although pure graphene macroscopic structures have been used as SC electrodes, their energy density is moderate. In order to improve the energy density of the graphene-based electrodes, CPs were added into the graphenebased electrodes. ${ }^{[120,125-129]}$ Using a vacuum filtration method, RGO/PANInanofibers (NFs) films were prepared. ${ }^{[120]}$ RGO/PANI-NF film has a high conductivity of $5.5 \times 10^{2} \mathrm{~S} / \mathrm{m}$, which is about 10 times higher than that of pure PANI-NFs. These films can be cut into desired shape and directly used as SC electrodes without insulating binders or conducting additives due to the ability of these materials to support themselves mechanically, along with having a high amount of flexibility. The specific capacitance of RGO/PANI-NFs film SCs is $210 \mathrm{~F} / \mathrm{g}$, which is also much higher than the average value of PANI-NF and RGO films (145 F/g). The internal resistance of RGO/PANI-NFs film SCs is much smaller than that of pure PANI-NFs SCs. The high conductivity of RGO/PANI-NFs film accelerated the charge-transfer during the discharging process, the RGO/PANI-NFs film maintained a higher capacitance of 94\% (197 $\mathrm{F} / \mathrm{g}$ ) as current was increased from 0.3 to $3 \mathrm{~A} / \mathrm{g}$ as against that of the PANI-NF film $(86 \%)$ in the same current range. However, when current was increased beyond $6 \mathrm{~A} / \mathrm{g}$, the specific capacitance of the RGO/PANI-NFs film was measured to be slightly lower than that of the PANI-NF film due to the limitations of the electrolyte. The electrochemical stability of the RGO/PANINFs film was improved by the addition of RGO sheets, which act as the frameworks for sustaining PANI-NFs, preventing the fibers from severely swelling and shrinking during cycling. Flexible graphene/PANI-NFs hybrid paper can also be prepared via a facile and fast two-step route composed of electrostatic adsorption between negatively-charged poly(sodium 4styrenesulfonate) (PSS) mediated graphene sheets (PSS-GS) and positivelycharged PANI NFs and the follow-up vacuum filtration of the as-prepared PSS- 
GS/PANI NFs suspension. ${ }^{[130]}$ Highly ordered, layered, PSS-GS/PANI composite papers have been produced where the PANI NFs are coated by PSSGS because of an electrostatic interaction. The highest specific capacitance of the composites reaches $301 \mathrm{~F} / \mathrm{g}$, owing to effective synergy between graphene and PANI NFs.

Based on LBL assembly, flexible multilayered RGO/PANI films can be obtained by alternate deposition of positively charged PANI and negatively charged GO nanosheets on a PET substrate, followed by chemical reduction of GO to RGO. ${ }^{[131]}$ The hybrid electrodes provide not only an improved electronic conductivity through the intimate contact with the rGO nanosheets, but also enhanced chemical stability during the charge-discharge process. A synergistic effect between an EDLC of rGO nanosheets and a pseudocapacitor of the $\mathrm{CP}$ PANI was exhibited for the flexible thin film SCs with excellent electrochemical performance. The electrochemical performance of the RGO/PANI hybrid film depends on the various parameters of the LBL assembly such as the number of bilayers and the post-thermal and chemical treatments that affect the degree of reduction of GO and PANI. RGO/PANI hybrid films possess an excellent gravimetric capacitance of $375.2 \mathrm{~F} / \mathrm{g}$ at a discharge current density of $0.5 \mathrm{~A} / \mathrm{g}$. The hybrid SC maintained its capacity up to $90.7 \%$ over 500 cycles at a high current density of $3.0 \mathrm{~A} / \mathrm{g}$. This study opens up the possibility for the production of diverse graphene-based hybrid nanocomposites that are promising for future flexible SCs.

Based on an in situ anodic electropolymerization of PANI on graphene paper, Cheng and co-workers prepared freestanding and flexible graphene/PANI composite papers. ${ }^{[127]}$ These hybrid films show a high capacitance of $233 \mathrm{~F} / \mathrm{g}$, reaching a $58 \%$ enhancement in comparison with the graphene paper $(147 \mathrm{~F} / \mathrm{g})$. These intriguing features make it quite a promising material as a freestanding electrode for flexible SCs. 
PPy is another one of most promising conductive polymers used in SCs. Multilayered nanoarchitectures of rGO sheets and PPy nanowires was prepared by combining liquid-air interface assembly with capillary force driven LBL assembly. ${ }^{[132]}$ In these multilayered films, the fibrous network of PPy nanowires, possessing high ionic accessibility, was interspersed with electrically conductive monolayers of highly aligned large sized rGO sheets as a series of current collectors. These multilayer film electrodes exhibit a specific capacitance of 165 $\mathrm{F} / \mathrm{g}$ at a $1 \mathrm{~A} / \mathrm{g}$ discharge current density even after 1000 electrochemical cycles. They maintain a high frequency capacitive response with a knee frequency close to $50 \mathrm{~Hz}$.

Pulsed electrodeposition utilizing well-separated short potential pulses has been employed to create flexible, uniform rGO/PPy hybrid films. ${ }^{[133]}$ Pulsed electrodeposition allows pyrrole monomers to diffuse into the pore space of the rGO between deposition pulses, and demonstrated a significant improvement in the uniformity of PPy coatings on rGO sheets. The addition of PPy still maintains the inherent flexibility of rGO films. The flexible SC based on rGO/PPy hybrid films displays a specific capacitance as high as $237 \mathrm{~F} / \mathrm{g}$ for a moderate total deposition time of only $120 \mathrm{~s}$, which is approximately four times higher than the blank scaffold, rGO films, due to the pseudocapacitive contribution of conformal redox-active polypyrrole. Furthermore, this flexible SC exhibited very high energy and power densities of $\sim 33 \mathrm{Wh} / \mathrm{kg}$ and $\sim 1184$ $\mathrm{W} / \mathrm{kg}$, respectively, at a scan rate of $10 \mathrm{mV} / \mathrm{s}$. This increase was attributed to the favorable nucleation of new polymer chains at defects on the graphene surface, which become less favorable as defect sites are occupied by existing polymer nanoparticles.

\subsection{Graphene/CNTs/conductive polymer for flexible Pseudo-capacitors}

The excellent mechanical properties of flexible rGO sheets and rigid CNT can synergistically release the differential strain of polymer chains during 
doping/dedoping processes for CPs, leading to excellent cyclic stability. ${ }^{[107,134]}$ Zhang et al distributed PPy/CNT composites between rGO sheets homogeneously by flow-assembly to prepare unique flexible films. ${ }^{[135]}$ $\mathrm{rGO} / \mathrm{PPy} / \mathrm{CNT}$ hybrid films exhibit good flexibility. In such a layered structure, the coaxial PPy/CNT nanocables can not only enlarge the space between rGO sheets but also provide pseudo-capacitance to enhance the total capacitance of electrodes. Multilayered $\mathrm{rGO} / \mathrm{PPy} / \mathrm{CNT}$ achieve high mass and volume specific capacitances of $211 \mathrm{~F} / \mathrm{g}$ and $122 \mathrm{~F} / \mathrm{cm}$ at a current density of $0.2 \mathrm{~A} / \mathrm{g}$, which is higher than those of the rGO film $\left(73 \mathrm{~F} / \mathrm{g}\right.$ and $\left.79 \mathrm{~F} / \mathrm{cm}^{2}\right)$ and PPy/CNT $(164 \mathrm{~F} / \mathrm{g}$ and $67 \mathrm{~F} / \mathrm{cm}^{2}$ ). Significantly, the $\mathrm{rGO} / \mathrm{PPy} / \mathrm{CNT}$ electrode shows excellent cyclic stability (with only a 5\% capacity loss after 5000 cycles) due to the flexible GN layer and the rigid CNT core synergistically releasing the intrinsic differential strain of PPy chains during long-term charge/discharge cycles.

\section{Carbon/Metal Oxide Composite}

In addition to conductive polymers, previous studies demonstrate that energy density of SCs can be effectively improved by utilizing nanostructured MOs, such as $\mathrm{MnO}_{2}$ and $\mathrm{RuO}_{2}$, as Faradic electrodes at the cost of electrochemical stability. The high surface area of rGO sheets and CNTs make them promising support for the deposition of MOs. Furthermore, the electrons from the reversible oxidation-reduction of MOs can be effectively transported through rGO or CNT structure due to the high conductivity of rGO sheets and CNTs, which benefit the improve the power density of hybrid electrode materials. The high mechanical properties of CNTs and rGO sheets endow the hybrid film electrodes with good flexibility.

\subsection{CNT/Metal Oxide Composite Flexible Pseudo-supercapacitors}

Lee et al prepare flexible CNT/MnOx hybrid sheets by electrodepositing MnOx onto CNT sheets (Figure 10a and b), pulled directly from MWCNT 
forests. ${ }^{[136]}$ The morphology and microstructure of the CNT/MnOx hybrid sheets varied substantially as the electrodeposition time was increased. Both the specific capacitances and rate capabilities depend on the average size of the MnOx nanoparticles on the CNTs, as indicated in CV and charge/discharge curves (Figure 10c and d). The resulting CNT/MnOx hybrid sheets have high specific capacitances of $1250 \mathrm{~F} / \mathrm{g}$, high charge/discharge rate capabilities, and excellent cyclic stability. These properties are ascribed to a synergistic combination of large surface area and good electron-transport capabilities of the MnOx nanoparticles with the good conductivity of the CNT sheets. Flexible high frequency response $\mathrm{SCs}$ can be fabricated using freestanding SWCNT/ $\mathrm{MnO}_{2}$ electrodes, which was obtained by a facile and scalable asymmetric in situ deposition method to incorporate $\mathrm{MnO}_{2}$ nanoparticles in vacuum filtrated SWCNT films. The as-prepared SCs showed a superior performance with specific capacitance of $529.8 \mathrm{~F} / \mathrm{g}$, energy density of 73.6 $\mathrm{Wh} / \mathrm{kg}$, power density of $14.6 \mathrm{Kw} / \mathrm{kg}$, excellent capacitance retention $(99.9 \%)$ after 2000 charge and discharge cycles, and high knee frequency of $1318 \mathrm{~Hz}$.

More recently, coating MO, and/or CNT composites on textiles, sponges, and papers have also been reported as feasible methods to prepare flexible electrodes for SCs. ${ }^{[11,137-139]}$ As mention in 3.1 section, highly conductive SWCNTs/cloth electrodes can be obtained by simple "dipping and drying" process using SWCNT ink. ${ }^{[1]]}$ To improve the energy density of the SWCNTs/cloth electrodes, $\mathrm{MnO}_{2}$ was uniformly electrodeposited on the SWCNTs. ${ }^{[1]}$ This was effective in significantly increasing the mass loading of the pseudocapacitor, while maintaining contact between SWNTs and providing a good electrical conduction path. The deposition is observed not only on the surfaces of the SWCNT/cotton but also inside the layers of cotton fibers. The capacitance with respect to the device increases by a factor of 24 after $\mathrm{MnO}_{2}$ deposition, reaching $0.41 \mathrm{~F} / \mathrm{cm}^{2}$. The specific capacitance when including the masses of both $\mathrm{CNT}$ and $\mathrm{MnO}_{2}$ increases by a factor of 4 with $\mathrm{MnO}_{2}$ deposition. 
In addition, this group reports a new nanostructure based on a conductive CNTtextile fiber network, where $\mathrm{MnO}_{2}$ nanoflowers were conformally electrodeposited onto CNT-enabled conductive textile fibers. ${ }^{[137]}$ Such a porous structure effectively decreases the ion diffusion and charge transport resistance in the electrodes and also allows a large mass loading, up to $8.3 \mathrm{mg} / \mathrm{cm}^{2}$, which leads to a high areal capacitance of $2.8 \mathrm{~F} / \mathrm{cm}^{2}$ at a scan rate of $0.05 \mathrm{mV} / \mathrm{s}$. In addition to the textile composite, this group also used sponges as support substrates to fabricate nanostructured $\mathrm{MnO}_{2} / \mathrm{CNT} /$ sponge hybrid electrodes. ${ }^{[138]}$ The SCs based on these hybrid electrodes exhibited high specific power and energy densities of $63 \mathrm{~kW} / \mathrm{kg}$ and $31 \mathrm{Wh} / \mathrm{kg}$, respectively. The $\mathrm{MnO}_{2} / \mathrm{CNT} /$ sponge SCs show only $4 \%$ of degradation after 10000 cycles at a charge-discharge specific current of $5 \mathrm{~A} / \mathrm{g}$. In addition, Kim et al reported that flexible paper-based SCs with increased specific capacitance and specific energy can be fabricated by depositing $\mathrm{MnO}_{2}$ on CNT-coated papers. The $\mathrm{MnO}_{2} / \mathrm{CNT} /$ paper SCs showed a high specific capacitance of $540 \mathrm{~F} / \mathrm{g}{ }^{[139]}$ Specific energy and specific power densities were determined to be $20 \mathrm{Wh} / \mathrm{kg}$ and $1.5 \mathrm{~kW} / \mathrm{kg}$, respectively at current density of $5 \mathrm{~A} / \mathrm{g}$

Using other CNT structures as templates, flexible $\mathrm{CNT} / \mathrm{MnO}_{2} \mathrm{SC}$ electrodes were also prepared. For example, flexible carbon fabric (CF)-CNT array (CNTA) $/ \mathrm{MnO}_{2}$ hybrid electrodes with a 3D porous structure are prepared by the electrochemical deposition of $\mathrm{MnO}_{2}$ onto CNTA. ${ }^{[140]}$ The maximum specific capacitance of CF-CNTA/ $/ \mathrm{MnO}_{2}$ electrodes is up to $740 \mathrm{~F} / \mathrm{g}$ at the scan rate of 2 $\mathrm{mV} / \mathrm{s}$. CF-CNTA/ $/ \mathrm{MnO}_{2}$ achieved a high retention of $70 \%$ with a scan rate range of 2-200 mV/s due to the $3 \mathrm{D}$ structures.

Based on SWCNT inks and an off-the-shelf inkjet printer, Zhou et al successfully printed SWCNT thin film electrodes on flexible substrates and cloth fabrics with features of controlled pattern geometry, location, thickness, and tunable electrical conductivity. ${ }^{[141]}$ Then, $\mathrm{RuO}_{2}$ in an isopropanol alcohol (IPA) suspension dispersed on the printed SWNT films until a reasonable 
nanowire density was achieved. The $\mathrm{SWCNT} / \mathrm{RuO}_{2}$ hybrid films were then sandwiched together with a piece of printable polymer electrolyte to form flexible and wearable SCs. Owing to the addition of $\mathrm{RuO}_{2}$, the performance of the SWCNT/RuO $\mathrm{Ru}_{2}$ hybrid film SC was significantly improved in terms of its specific capacitance of $138 \mathrm{~F} / \mathrm{g}$, power density of $96 \mathrm{~kW} / \mathrm{kg}$, and energy density of $18.8 \mathrm{Wh} / \mathrm{kg}$. Furthermore, the knee frequency of the hybrid thin film electrodes can reach $1,500 \mathrm{~Hz}$, which is much higher than the knee frequency of the bare SWCNT electrodes $(\sim 158 \mathrm{~Hz})$.

Owing to the unique properties of MO nanowires, such as high aspect ratio and short diffusion path length for ions, they can provide high surface area, fast charge/discharge, and facial redox reactions, and thus can be one of good candidates for electrochemical capacitors. As is well known, $\operatorname{In}_{2} \mathrm{O}_{3}$ is a wide band gap material $(3.57 \mathrm{eV})$, which has been widely applied in the transparent electronics. Zhou et al improved the conductivity of $\mathrm{In}_{2} \mathrm{O}_{3}$ electrodes by integrating $\mathrm{In}_{2} \mathrm{O}_{3}$ together with SWCNTs to form hybrid nanostructured films

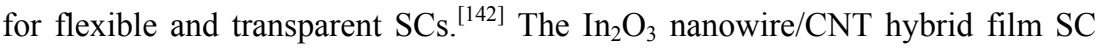
exhibits high specific capacitance of $64 \mathrm{~F} / \mathrm{g}$, high power density of $7.48 \mathrm{~kW} / \mathrm{kg}$ and a good retention of capacity ( $88 \%$ after 500 cycles). An alternative approach to improve energy density is to develop asymmetric SCs (ASCs). ASCs can make use of the different potential windows of the two electrodes to increase the maximum operation voltage of the aqueous electrolyte in the device. Zhou et al has developed an easy and efficient method to prepare hybrid nanostructured thin film electrodes by using two different transition-metal-oxide nanowires, including $\mathrm{MnO}_{2}$ nanowires and $\mathrm{In}_{2} \mathrm{O}_{3}$ nanowires, together with SWCNTs and fabricated hybrid nanostructured $\mathrm{ASCs} .{ }^{[143]}$ In this asymmetric $\mathrm{SC}$, a $\mathrm{MnO}_{2}$ nanowire/SWCNT hybrid film served as the positive electrode, while a $\operatorname{In}_{2} \mathrm{O}_{3}$ nanowire/SWCNT hybrid film functioned as the negative electrode with a neutral electrolyte. These hybrid nanostructured films obtained from a filtration method possess mechanical flexibility, uniform layered structures, and 
mesoporous surface morphology. The mass balance between two electrodes has been optimized to obtain stable electrochemical behavior during chargingdischarging cycles in a $2 \mathrm{~V}$ potential window. The optimized hybrid nanostructured ASCs exhibited a superior device performance with specific capacitance of $184 \mathrm{~F} / \mathrm{g}$, energy density of $25.5 \mathrm{Wh} / \mathrm{kg}$, power density of 50.3 $\mathrm{kW} / \mathrm{kg}$ and columbic efficiency of $90 \%$.

In addition to $\mathrm{MnO}_{2}$ and $\mathrm{In}_{2} \mathrm{O}_{3}$, other MOs were also developed to improve the performance of the electrodes. Generally, vanadium oxides offer high pseudo-capacitance but limited electrical conductivity and specific surface area. To overcome this issue, smooth nanostructured vanadium oxide with various thicknesses can be coated uniformly on the surface of porous MWCNT electrodes by atomic layer deposition. ${ }^{[144]}$ The as-prepared binder-free flexible hybrid electrodes have controlled porosity, greatly improved electrical conductivity and cycle stability. Remarkable capacitance of up to $\sim 1550 \mathrm{~F} / \mathrm{g}$ per active mass of the vanadium oxide and up to $\sim 600 \mathrm{~F} / \mathrm{g}$ per mass of the composite electrode MWCNT/vanadium oxide electrodes were achieved, significantly exceeding specific capacitance of commercially used activated carbons (100$150 \mathrm{~F} / \mathrm{g}$ ). In addition, flexible $\mathrm{CNT} / \mathrm{TiO}_{2}$ hybrid electrodes can be obtained by coating CNT networks on the $\mathrm{TiO}_{2}$ nanotubes. ${ }^{[145]}$ The specific capacitance of $\mathrm{CNT} / \mathrm{TiO}_{2}$ hybrid electrodes can be enhanced by about $30 \%$ as compared to pure carbon-nanotube electrodes.

\subsection{Graphene/MO composite flexible Pseudo-capacitors}

3D macroporous RGO electrodes can be fabricated by replicating and embossing techniques using polystyrene colloidal particles as a sacrificial template. ${ }^{[146]}$ For further improvement of capacitance, a thin layer of $\mathrm{MnO}_{2}$ was additionally deposited onto the RGO surface in the $3 \mathrm{D}$ structure. The macroporous hybrid structure endows a large surface area and facilitates fast ionic transport within the electrode while preserving decent electronic 
conductivity. These $\mathrm{MnO}_{2} / \mathrm{RGO}$ hybrid composite electrodes exhibit a high specific capacitance of $389 \mathrm{~F} / \mathrm{g}$ at $1 \mathrm{~A} / \mathrm{g}$ and $97.7 \%$ capacitance retention upon a current increase to $35 \mathrm{~A} / \mathrm{g}$. Moreover, when the $\mathrm{MnO}_{2} / \mathrm{RGO}$ composite electrode was asymmetrically assembled with an RGO macroporous electrode, the resulting SCs possess energy density of $44 \mathrm{Wh} / \mathrm{kg}$, power density of $25 \mathrm{~kW} / \mathrm{kg}$, and excellent cycle life. Flexible $\mathrm{RGO} / \mathrm{MnO}_{2}$ hybrid papers have successfully fabricated via a simple three-step route: preparation of $\mathrm{GO} / \mathrm{MnO}_{2}$ composite dispersion, subsequent vacuum filtration of the composite dispersion to achieve $\mathrm{GO} / \mathrm{MnO}_{2}$ hybrid paper, and finally thermal reduction of the $\mathrm{GO} / \mathrm{MnO}_{2}$ to generate $\mathrm{RGO} / \mathrm{MnO}_{2}$ hybrid paper. ${ }^{[147]}$ These $\mathrm{RGO} / \mathrm{MnO}_{2}$ hybrid papers can be used as a binder-free and flexible electrode material for SCs. The specific capacitance of the hybrid paper with the $\mathrm{MnO}_{2}$ weight ratio of $24 \%$ reaches 256 F/g at a current density of $500 \mathrm{~mA} / \mathrm{g}$ and also shows good cycle stability.

Solution-based coating and printing techniques have been recently exploited to make conductive CNT-based papers and textiles as electrodes and/or current collectors for SCs. ${ }^{[11]}$ Using a similar concept, Bao et al demonstrate that rGO nanosheets can be conformably coated from solution on the fiber surfaces of $3 \mathrm{D}$ porous textile support structures, serving as a conductive support with large surface area for the subsequent deposition of nanoscale $\mathrm{MnO}_{2}$ particles, as shown in Figure 10e and $\mathrm{f}^{[148]} 3 \mathrm{D}$ porous textiles ensure the high loading of active electrode materials and facilitate the access of electrolytes to those materials. As the scan rate increases from 50 to $100 \mathrm{mV} / \mathrm{s}$, the peak current increases and the shape of $\mathrm{CV}$ curves shows some distortions from an ideal capacitor, possibly due to increasing overpotentials from ion transport between the electrolyte and $\mathrm{MnO}_{2}$, as show in Figure $10 \mathrm{~g}$. The hybrid $\mathrm{rGO} / \mathrm{MnO}_{2}$-based textile possesses a high-capacitance performance with specific capacitance up to $315 \mathrm{~F} / \mathrm{g}$ (Figure 10h). To improve the power density and cycling of graphene/ $\mathrm{MnO}_{2}$-based textile, this group developed a "conductive wrapping" method to greatly improve the $\mathrm{SC}$ performance of $\mathrm{rGO} / \mathrm{MnO}_{2}$-based 
nanostructured electrodes. ${ }^{[149]}$ Owing to $3 \mathrm{D}$ conductive wrapping of $\mathrm{rGO} / \mathrm{MnO}_{2}$ nanostructures with CNTs or $\mathrm{CP}$, specific capacitance of the electrodes (considering total mass of active materials) has substantially increased by $20 \%$ and $45 \%$, respectively, with values as high as $380 \mathrm{~F} / \mathrm{g}$ achieved. Moreover, these ternary composite electrodes have also exhibited excellent cycling performance with $>95 \%$ capacitance retention over 3000 cycles.

Apart from $\mathrm{MnO}_{2}$, other MOs also exhibit the capacity of improving the energy density of graphene-based SCs. Well-designed flexible and free-standing $\mathrm{Co}_{3} \mathrm{O}_{4} / \mathrm{RGO} / \mathrm{CNT}$ hybrid paper electrodes prepared by one-step hydrothermal method exhibit a specific capacitance of $378 \mathrm{~F} / \mathrm{g}$ at $2 \mathrm{~A} / \mathrm{g}$ and $297 \mathrm{~F} / \mathrm{g}$ at $8 \mathrm{~A} / \mathrm{g}$ with excellent electrochemical stability. ${ }^{[150]}$ Zhang et al prepared $3 \mathrm{D}$ graphene/ $\mathrm{NiO}$ networks by electrochemically depositing $\mathrm{NiO}$ on the $3 \mathrm{D}$ graphene networks prepared by CVD with $\mathrm{Ni}$ foam as a sacrificial template. The graphene/ $\mathrm{NiO}$ composite electrode exhibits a high specific capacitance of 816 $\mathrm{F} / \mathrm{g}$ at $5 \mathrm{mV} / \mathrm{s}$ along with a good rate capability. Taking into account the easy hydrothermal growth of $\mathrm{ZnO}$ on various substrates, especially on graphene films, tailoring nanostructures, and its pseudo-capacitance behavior, Huang et al propose a $3 \mathrm{D}$ structure of vertical alligned $\mathrm{ZnO}$ nanorods sandwiched between RGO films as flexible SC electrodes. $\mathrm{ZnO}$ nanorods in the electrodes prevent aggregation of RGO sheets and help in forming a porous structure. A specific capacitance of $51.6 \mathrm{~F} / \mathrm{g}$ at the scan rate of $10 \mathrm{mV} / \mathrm{s}$ is achieved for the $\mathrm{RGO} / \mathrm{ZnO}$ nanorods/RGO sandwich. As a novel composite of one-atom-thick sheets, Co-Al layered double hydroxide nanosheets can be used as supercacitors. Flexible multilayer hybrid films of Co-Al layered double hydroxide nanosheets (Co-Al LDH-NS) and GO were fabricated through LBL assembly. The Co Al LDHNS/GO multilayer films exhibited a high specific capacitance of $880 \mathrm{~F} / \mathrm{g}$ and area capacitance of $70 \mathrm{~F} / \mathrm{m}^{2}$ under the scan rate of $5 \mathrm{mV} / \mathrm{s}$. In addition, the film exhibited good cycle stability over 2000 cycles. After reduction of GO to RGO, 
the specific capacitance and area capacitance were largely increased up to 1204 $\mathrm{F} / \mathrm{g}$ and $90 \mathrm{~F} / \mathrm{m}^{2}$.

\subsection{Graphene/CNT/MO Composite flexible Pseudo-capacitors}

Graphene has been widely considered as an ideal substrate for ultrathin coating of functional materials due to its unique structural and electrical properties, while the uniformly interconnected CNTs network is beneficial for electronic and ionic transport due to its high conductivity and porousity. While $\mathrm{MnO}_{2}$ has attracted much attention in $\mathrm{SC}$ applications due to its high capacitance due to pseudo-capacitance, its low conductivity significantly reduces the overall power density of devices. To fully utilize their own advantages of graphene, CNTs and $\mathrm{MnO}_{2}$, Liu and co-workers present a novel method to fabricate conductive, highly flexible, and robust film SC electrodes based on $\mathrm{rGO} / \mathrm{MnO}_{2} / \mathrm{CNTs}$ nanocomposites. Synergistic effects from both $\mathrm{rGO}$ and CNTs were utilised as the rGO provides a high-surface-area substrate for direct growth of $\mathrm{MnO}_{2}$ nanoparticles and CNTs provide electron conductance and mechanical reinforcement. The synergistic effects from rGO, CNTs, and $\mathrm{MnO}_{2}$ deliver outstanding mechanical properties (tensile strength of $48 \mathrm{MPa}$ ) and superior electrochemical activity that were not achieved by any of these components individually. These flexible electrodes allow for a high specific capacitance (372 $\mathrm{F} / \mathrm{g}$ ) with excellent rate capability for SCs without the need of current collectors and binders. The film can also be wound around $0.5 \mathrm{~mm}$ diameter rods for fabricating full cells with high performance, showing significant potential in flexible energy storage devices.

\section{Perspectives}

Although some great progresses have been achieved in both preparation of flexible SC electrodes and assembly of flexible SC based on these electrodes, much work remains to be done in the preparation of these electrodes. Further 
work is needed for controllable assembly of electrodes and devices, improvement of properties, and extended of practical applications.

Although various flexible SC electrodes have been successfully fabricated by different methods, such as vacuum filtration, solution-based coating and printing techniques, and high specific capacitance have been obtained, the overall capacitance is much smaller than the practical requirement due to the limited mass and area of electrodes. Preparation of flexible SC with high capacitance should be considered for the practical application of flexible supercpacitors. Currently various flexible functional devices have been reported. In future, flexible SCs with controlled design should be prepared to match and power these functional devices, even integrate the flexible SCs with these functional devices into fully flexible devices. The flexibility of SC electrodes should also be improved by controlling the structure of electrode materials. The measurement methods for the flexibility and eletrochemical performance of flexible supercapacitors are not well standardized and as a result it is difficult to assess the true performance reported in the literature. Test methods for flexible ultracapacitors should be well developed.

Graphene and CNT have high theoretical SSA. However, the practical SSA of pure graphene and/or CNT SC electrodes is much smaller than the theoretical value due to their structure, in which not all their surface area is electrochemically accessible when in contact with electrolyte. In further work, the SSA utilisation of graphene and CNT in the flexible electrodes should be improved by controlling their porous structure to increase the energy density of the electrodes. Graphene- and CNT-based hybrid electrodes would be optimized by controlling their compositions, morphology, micro-structure to enhance their power density.

Assembly of devices is another issue that affects the performance and flexibility of SCs. Conventional flexible SC devices, as show in Figure 1, can not meet the requirement of highly flexible SCs. SCs with different design and 
structure should be explored. The SCs will move to compact, flexible, lightweight and low-cost direction. 


\section{References}

[1] Zhang, L. L.; Zhao, X. S. Chem. Soc. Rev. 2009, 38, 2520-2531.

[2] Zhang, Y.; Feng, H.; Wu, X. B.; Wang, L. Z.; Zhang, A. Q.; Xia, T. C.; Dong, H. C.; Li, X. F.; Zhang, L. S. Int. J. Hydrogen Energy 2009, 34, 4889-4899.

[3] Simon, P.; Gogotsi, Y. Nat. Mater. 2008, 7, 845-854.

[4] Winter, M.; Brodd, R. J. Chem. Rev. 2004, 104, 4245-4269.

[5] Talpone, J. I.; Puleston, P. F.; More, J. J.; Grino, R.; Cendoya, M. G. Int. J. Hydrogen Energy 2012, 37, 10346-10353.

[6] Kaempgen, M.; Chan, C. K.; Ma, J.; Cui, Y.; Gruner, G. Nano Lett. 2009, 9, 1872-1876.

[7] Niu, C. M.; Sichel, E. K.; Hoch, R.; Moy, D.; Tennent, H. Appl. Phys. Lett. 1997, 70, 1480-1482.

[8] Frackowiak, E.; Metenier, K.; Bertagna, V.; Beguin, F. Appl. Phys. Lett. 2000, 77, 2421-2423.

[9] Hu, L. B.; Choi, J. W.; Yang, Y.; Jeong, S.; La Mantia, F.; Cui, L. F.; Cui, Y. Proc. Natl Acad. Sci. USA 2009, 106, 21490-21494.

[10] Kang, Y. J.; Chung, H.; Han, C. H.; Kim, W. Nanotechnology 2012, 23.

[11] Hu, L. B.; Pasta, M.; La Mantia, F.; Cui, L. F.; Jeong, S.; Deshazer, H. D.; Choi, J. W.; Han, S. M.; Cui, Y. Nano Lett. 2010, 10, 708-714.

[12] Hu, S.; Rajamani, R.; Yu, X. Appl. Phys. Lett. 2012, 100.

[13] Pushparaj, V. L.; Shaijumon, M. M.; Kumar, A.; Murugesan, S.; Ci, L.; Vajtai, R.; Linhardt, R. J.; Nalamasu, O.; Ajayan, P. M. Proc. Natl Acad. Sci. USA 2007, 104, 13574-13577.

[14] Ma, W. J.; Song, L.; Yang, R.; Zhang, T. H.; Zhao, Y. C.; Sun, L. F.; Ren, Y.; Liu, D. F.; Liu, L. F.; Shen, J.; Zhang, Z. X.; Xiang, Y. J.; Zhou, W. Y.; Xie, S. S. Nano Lett. 2007, 7, 2307-2311.

[15] Ma, W. J.; Liu, L. Q.; Zhang, Z.; Yang, R.; Liu, G.; Zhang, T. H.; An, X. F.; Yi, X. S.; Ren, Y.; Niu, Z. Q.; Li, J. Z.; Dong, H. B.; Zhou, W. Y.; Ajayan, P. M.; Xie, S. S. Nano Lett. 2009, 9, 2855-2861.

[16] Niu, Z. Q.; Zhou, W. Y.; Chen, J.; Feng, G. X.; Li, H.; Ma, W. J.; Li, J. Z.; Dong, H. B.; Ren, Y.; Zhao, D.; Xie, S. S. Energy Environ. Sci. 2011, 4, 1440-1446.

[17] Niu, Z. Q.; Zhou, W. Y.; Chen, J.; Feng, G. X.; Li, H.; Hu, Y. S.; Ma, W. J.; Dong, H. B.; Li, J. Z.; Xie, S. S. Small 2013, 9, 518-524.

[18] Chen, J.; Minett, A. I.; Liu, Y.; Lynam, C.; Sherrell, P.; Wang, C.; Wallace, G. G. Adv. Mater. 2008, 20, 566-570.

[19] Chen, J.; Wang, J. Z.; Minett, A. I.; Liu, Y.; Lynam, C.; Liu, H. K.; Wallace, G. G. Energy Environ. Sci. 2009, 2, 393-396. 
[20] Xu, G. H.; Zheng, C.; Zhang, Q.; Huang, J. Q.; Zhao, M. Q.; Nie, J. Q.; Wang, X. H.; Wei, F. Nano Research 2011, 4, 870-881.

[21] Bolotin, K. I.; Ghahari, F.; Shulman, M. D.; Stormer, H. L.; Kim, P. Nature 2009, 462, 196-199.

[22] Chen, C. F.; Park, C. H.; Boudouris, B. W.; Horng, J.; Geng, B. S.; Girit, C.; Zettl, A.; Crommie, M. F.; Segalman, R. A.; Louie, S. G.; Wang, F. Nature 2011, 471, 617-620.

[23] Du, X.; Skachko, I.; Duerr, F.; Luican, A.; Andrei, E. Y. Nature 2009, 462, 192-195.

[24] Garaj, S.; Hubbard, W.; Reina, A.; Kong, J.; Branton, D.; Golovchenko, J. A. Nature 2010, 467, 190-U73.

[25] Gomes, K. K.; Mar, W.; Ko, W.; Guinea, F.; Manoharan, H. C. Nature 2012, 483, 306-310.

[26] Huang, P. Y.; Ruiz-Vargas, C. S.; van der Zande, A. M.; Whitney, W. S.; Levendorf, M. P.; Kevek, J. W.; Garg, S.; Alden, J. S.; Hustedt, C. J.; Zhu, Y.; Park, J.; McEuen, P. L.; Muller, D. A. Nature 2011, 469, 389-392.

[27] Kim, K. S.; Zhao, Y.; Jang, H.; Lee, S. Y.; Kim, J. M.; Ahn, J. H.; Kim, P.; Choi, J. Y.; Hong, B. H. Nature 2009, 457, 706-710.

[28] Lui, C. H.; Liu, L.; Mak, K. F.; Flynn, G. W.; Heinz, T. F. Nature 2009, 462, 339-341.

[29] Meyer, J. C.; Geim, A. K.; Katsnelson, M. I.; Novoselov, K. S.; Booth, T. J.; Roth, S. Nature 2007, 446, 60-63.

[30] Novoselov, K. S.; Geim, A. K.; Morozov, S. V.; Jiang, D.; Katsnelson, M. I.; Grigorieva, I. V.; Dubonos, S. V.; Firsov, A. A. Nature 2005, 438, $197-$ 200.

[31] Son, Y. W.; Cohen, M. L.; Louie, S. G. Nature 2006, 444, 347-349.

[32] Stankovich, S.; Dikin, D. A.; Dommett, G. H. B.; Kohlhaas, K. M.; Zimney, E. J.; Stach, E. A.; Piner, R. D.; Nguyen, S. T.; Ruoff, R. S. Nature 2006, 442, 282-286.

[33] Zhang, Y. B.; Tan, Y. W.; Stormer, H. L.; Kim, P. Nature 2005, 438, 201204.

[34] Berger, C.; Song, Z. M.; Li, X. B.; Wu, X. S.; Brown, N.; Naud, C.; Mayou, D.; Li, T. B.; Hass, J.; Marchenkov, A. N.; Conrad, E. H.; First, P. N.; de Heer, W. A. Science 2006, 312, 1191-1196.

[35] Bostwick, A.; Speck, F.; Seyller, T.; Horn, K.; Polini, M.; Asgari, R.; MacDonald, A. H.; Rotenberg, E. Science 2010, 328, 999-1002.

[36] Bunch, J. S.; van der Zande, A. M.; Verbridge, S. S.; Frank, I. W.; Tanenbaum, D. M.; Parpia, J. M.; Craighead, H. G.; McEuen, P. L. Science 2007, 315, 490-493.

[37] Dimiev, A.; Kosynkin, D. V.; Sinitskii, A.; Slesarev, A.; Sun, Z. Z.; Tour, J. M. Science 2011, 331, 1168-1172. 
[38] Elias, D. C.; Nair, R. R.; Mohiuddin, T. M. G.; Morozov, S. V.; Blake, P.; Halsall, M. P.; Ferrari, A. C.; Boukhvalov, D. W.; Katsnelson, M. I.; Geim, A. K.; Novoselov, K. S. Science 2009, 323, 610-613.

[39] Geim, A. K. Science 2009, 324, 1530-1534.

[40] Girit, C. O.; Meyer, J. C.; Erni, R.; Rossell, M. D.; Kisielowski, C.; Yang, L.; Park, C. H.; Crommie, M. F.; Cohen, M. L.; Louie, S. G.; Zettl, A. Science 2009, 323, 1705-1708.

[41] Lin, Y. M.; Valdes-Garcia, A.; Han, S. J.; Farmer, D. B.; Meric, I.; Sun, Y. N.; Wu, Y. Q.; Dimitrakopoulos, C.; Grill, A.; Avouris, P.; Jenkins, K. A. Science 2011, 332, 1294-1297.

[42] Miller, J. R.; Outlaw, R. A.; Holloway, B. C. Science 2010, 329, $1637-$ 1639.

[43] Nair, R. R.; Blake, P.; Grigorenko, A. N.; Novoselov, K. S.; Booth, T. J.; Stauber, T.; Peres, N. M. R.; Geim, A. K. Science 2008, 320, 1308-1308.

[44] Seol, J. H.; Jo, I.; Moore, A. L.; Lindsay, L.; Aitken, Z. H.; Pettes, M. T.; Li, X. S.; Yao, Z.; Huang, R.; Broido, D.; Mingo, N.; Ruoff, R. S.; Shi, L. Science 2010, 328, 213-216.

[45] Wei, Z. Q.; Wang, D. B.; Kim, S.; Kim, S. Y.; Hu, Y. K.; Yakes, M. K.; Laracuente, A. R.; Dai, Z. T.; Marder, S. R.; Berger, C.; King, W. P.; de Heer, W. A.; Sheehan, P. E.; Riedo, E. Science 2010, 328, 1373-1376.

[46] Westervelt, R. M. Science 2008, 320, 324-325.

[47] Zhao, L. Y.; He, R.; Rim, K. T.; Schiros, T.; Kim, K. S.; Zhou, H.; Gutierrez, C.; Chockalingam, S. P.; Arguello, C. J.; Palova, L.; Nordlund, D.; Hybertsen, M. S.; Reichman, D. R.; Heinz, T. F.; Kim, P.; Pinczuk, A.; Flynn, G. W.; Pasupathy, A. N. Science 2011, 333, 999-1003.

[48] Zhu, Y. W.; Murali, S.; Stoller, M. D.; Ganesh, K. J.; Cai, W. W.; Ferreira, P. J.; Pirkle, A.; Wallace, R. M.; Cychosz, K. A.; Thommes, M.; Su, D.; Stach, E. A.; Ruoff, R. S. Science 2011, 332, 1537-1541.

[49] Geim, A. K.; Novoselov, K. S. Nat. Mater. 2007, 6, 183-191.

[50] Morozov, S. V.; Novoselov, K. S.; Katsnelson, M. I.; Schedin, F.; Elias, D. C.; Jaszczak, J. A.; Geim, A. K. Phys. Rev. Lett. 2008, 100.

[51] Durkop, T.; Getty, S. A.; Cobas, E.; Fuhrer, M. S. Nano Lett. 2004, 4, 3539.

[52] Stoller, M. D.; Park, S. J.; Zhu, Y. W.; An, J. H.; Ruoff, R. S. Nano Lett. 2008, 8, 3498-3502.

[53] Lee, C.; Wei, X. D.; Kysar, J. W.; Hone, J. Science 2008, 321, 385-388.

[54] Balandin, A. A.; Ghosh, S.; Bao, W. Z.; Calizo, I.; Teweldebrhan, D.; Miao, F.; Lau, C. N. Nano Lett. 2008, 8, 902-907.

[55] Zhu, Y. W.; Murali, S.; Cai, W. W.; Li, X. S.; Suk, J. W.; Potts, J. R.; Ruoff, R. S. Adv. Mater. 2010, 22, 5226-5226.

[56] Huang, Y.; Liang, J. J.; Chen, Y. S. Small 2012, 8, 1805-1834. 
[57] Novoselov, K. S.; Geim, A. K.; Morozov, S. V.; Jiang, D.; Zhang, Y.; Dubonos, S. V.; Grigorieva, I. V.; Firsov, A. A. Science 2004, 306, 666669.

[58] Li, X. S.; Cai, W. W.; An, J. H.; Kim, S.; Nah, J.; Yang, D. X.; Piner, R.; Velamakanni, A.; Jung, I.; Tutuc, E.; Banerjee, S. K.; Colombo, L.; Ruoff, R. S. Science 2009, 324, 1312-1314.

[59] Zhang, B.; Lee, W. H.; Piner, R.; Kholmanov, I.; Wu, Y. P.; Li, H. F.; Ji, H. X.; Ruoff, R. S. ACS Nano 2012, 6, 2471-2476.

[60] Wu, W.; Jauregui, L. A.; Su, Z. H.; Liu, Z. H.; Bao, J. M.; Chen, Y. P.; Yu, Q. K. Adv. Mater. 2011, 23, 4898-4903.

[61] Wassei, J. K.; Mecklenburg, M.; Torres, J. A.; Fowler, J. D.; Regan, B. C.; Kaner, R. B.; Weiller, B. H. Small 2012, 8, 1415-1422.

[62] [Wang, Y.; Zheng, Y.; Xu, X. F.; Dubuisson, E.; Bao, Q. L.; Lu, J.; Loh, K. P. ACS Nano 2011, 5, 9927-9933.

[63] Tao, L.; Lee, J.; Chou, H.; Holt, M.; Ruoff, R. S.; Akinwande, D. ACS Nano 2012, 6, 2319-2325.

[64] Robertson, A. W.; Warner, J. H. Nano Lett. 2011, 11, 1182-1189.

[65] Rasool, H. I.; Song, E. B.; Mecklenburg, M.; Regan, B. C.; Wang, K. L.; Weiller, B. H.; Gimzewski, J. K. J. Am. Chem. Soc. 2011, 133, 1253612543.

[66] Rasool, H. I.; Song, E. B.; Allen, M. J.; Wassei, J. K.; Kaner, R. B.; Wang, K. L.; Weiller, B. H.; Gimzewski, J. K. Nano Lett. 2011, 11, 251-256.

[67] He, R.; Zhao, L. Y.; Petrone, N.; Kim, K. S.; Roth, M.; Hone, J.; Kim, P.; Pasupathy, A.; Pinczuk, A. Nano Lett. 2012, 12, 2408-2413.

[68] Han, G. H.; Gunes, F.; Bae, J. J.; Kim, E. S.; Chae, S. J.; Shin, H. J.; Choi, J. Y.; Pribat, D.; Lee, Y. H. Nano Lett. 2011, 11, 4144-4148.

[69] Gao, L. B.; Ren, W. C.; Zhao, J. P.; Ma, L. P.; Chen, Z. P.; Cheng, H. M. Appl. Phys. Lett. 2010, 97.

[70] Gao, L.; Guest, J. R.; Guisinger, N. P. Nano Lett. 2010, 10, 3512-3516.

[71] Reina, A.; Thiele, S.; Jia, X. T.; Bhaviripudi, S.; Dresselhaus, M. S.; Schaefer, J. A.; Kong, J. Nano Research 2009, 2, 509-516.

[72] Lahiri, J.; Miller, T.; Adamska, L.; Oleynik, II; Batzill, M. Nano Lett. 2011, 11, 518-522.

[73] Addou, R.; Dahal, A.; Sutter, P.; Batzill, M. Appl. Phys. Lett. 2012, 100.

[74] Li, X. S.; Cai, W. W.; Colombo, L.; Ruoff, R. S. Nano Lett. 2009, 9, 42684272.

[75] Chen, S. S.; Cai, W. W.; Piner, R. D.; Suk, J. W.; Wu, Y. P.; Ren, Y. J.; Kang, J. Y.; Ruoff, R. S. Nano Lett. 2011, 11, 3519-3525.

[76] Sutter, P. W.; Flege, J. I.; Sutter, E. A. Nat. Mater. 2008, 7, 406-411.

[77] Cui, X.; Zhang, C. Z.; Hao, R.; Hou, Y. L. Nanoscale 2011, 3, 2118-2126.

[78] Li, C.; Shi, G. Q. Nanoscale 2012, 4, 5549-5563. 
[79] Dikin, D. A.; Stankovich, S.; Zimney, E. J.; Piner, R. D.; Dommett, G. H. B.; Evmenenko, G.; Nguyen, S. T.; Ruoff, R. S. Nature 2007, 448, $457-$ 460.

[80] Chen, D.; Tang, L. H.; Li, J. H. Chem. Soc. Rev. 2010, 39, 3157-3180.

[81] Dreyer, D. R.; Park, S.; Bielawski, C. W.; Ruoff, R. S. Chem. Soc. Rev. 2010, 39, 228-240.

[82] Li, D.; Muller, M. B.; Gilje, S.; Kaner, R. B.; Wallace, G. G. Nat. Nanotechnol. 2008, 3, 101-105.

[83] Niu, Z. Q.; Chen, J.; Hng, H. H.; Ma, J.; Chen, X. D. Adv. Mater. 2012, 24, 4144-4150.

[84] Yang, X. W.; Zhu, J. W.; Qiu, L.; Li, D. Adv. Mater. 2011, 23, 2833-2838.

[85] Tai, Z. X.; Yan, X. B.; Xue, Q. J. J. Power Sources 2012, 213, 350-357.

[86] Chen, C. M.; Zhang, Q.; Huang, C. H.; Zhao, X. C.; Zhang, B. S.; Kong, Q. Q.; Wang, M. Z.; Yang, Y. G.; Cai, R.; Su, D. S. Chem. Commun. 2012, 48, 7149-7151.

[87] Gao, W.; Singh, N.; Song, L.; Liu, Z.; Reddy, A. L. M.; Ci, L. J.; Vajtai, R.; Zhang, Q.; Wei, B. Q.; Ajayan, P. M. Nat. Nanotechnol. 2011, 6, 496500 .

[88] El-Kady, M. F.; Strong, V.; Dubin, S.; Kaner, R. B. Science 2012, 335, 1326-1330.

[89] Zhang, L. L.; Zhao, X.; Stoller, M. D.; Zhu, Y. W.; Ji, H. X.; Murali, S.; Wu, Y. P.; Perales, S.; Clevenger, B.; Ruoff, R. S. Nano Lett. 2012, 12, 1806-1812.

[90] Biswas, S.; Drzal, L. T. Acs Applied Materials \& Interfaces 2010, 2, 2293 2300.

[91] Niu, Z. Q.; Du, J. J.; B., C. X.; Sun, Y. H.; Zhou, W. Y.; Hng, H. H.; Ma, J.; Chen, X. D.; Xie, S. S. Small 2012, 8, 3201-3208.

[92] Wang, G. K.; Sun, X.; Lu, F. Y.; Sun, H. T.; Yu, M. P.; Jiang, W. L.; Liu, C. S.; Lian, J. Small 2012, 8, 452-459.

[93] Yu, A. P.; Roes, I.; Davies, A.; Chen, Z. W. Appl. Phys. Lett. 2010, 96.

[94] Liu, W. W.; Yan, X. B.; Lang, J. W.; Peng, C.; Xue, Q. J. J. Mater. Chem. 2012, 22, 17245-17253.

[95] Tai, Z. X.; Yan, X. B.; Lang, J. W.; Xue, Q. J. J. Power Sources 2012, 199, 373-378.

[96] Kang, Y. R.; Li, Y. L.; Hou, F.; Wen, Y. Y.; Su, D. Nanoscale 2012, 4, 3248-3253.

[97] Peng, L. W.; Feng, Y. Y.; Lv, P.; Lei, D.; Shen, Y. T.; Li, Y.; Feng, W. J. Phys. Chem. C 2012, 116, 4970-4978.

[98] Lu, X. J.; Dou, H.; Gao, B.; Yuan, C. Z.; Yang, S. D.; Hao, L.; Shen, L. F.; Zhang, X. G. Electrochim. Acta 2011, 56, 5115-5121.

[99] Li, Y. F.; Liu, Y. Z.; Yang, Y. G.; Wang, M. Z.; Wen, Y. F. Appl. Phys. A 2012, 108, 701-707.

[100]Yu, D. S.; Dai, L. M. J. Phys. Chem. Lett. 2010, 1, 467-470. 
[101] Izadi-Najafabadi, A.; Yamada, T.; Futaba, D. N.; Yudasaka, M.; Takagi, H.; Hatori, H.; Iijima, S.; Hata, K. ACS Nano 2011, 5, 811-819.

[102] Obreja, V. V. N. Physica E-Low-Dimensional Systems \& Nanostructures 2008, 40, 2596-2605.

[103]Hyder, M. N.; Lee, S. W.; Cebeci, F. C.; Schmidt, D. J.; Shao-Horn, Y.; Hammond, P. T. ACS Nano 2011, 5, 8552-8561.

[104] Salvatierra, R. V.; Oliveira, M. M.; Zarbin, A. J. G. Chem. Mater. 2010, $22,5222-5234$.

[105]Meng, C. Z.; Liu, C. H.; Fan, S. S. Electrochem. Commun. 2009, 11, 186189.

[106] Mikhaylova, A. A.; Tusseeva, E. K.; Mayorova, N. A.; Rychagov, A. Y.; Volfkovich, Y. M.; Krestinin, A. V.; Khazova, O. A. Electrochim. Acta 2011, 56, 3656-3665.

[107] Yan, J.; Wei, T.; Fan, Z. J.; Qian, W. Z.; Zhang, M. L.; Shen, X. D.; Wei, F. J. Power Sources 2010, 195, 3041-3045.

[108] Wang, T.; Kiebele, A.; Ma, J.; Mhaisalkar, S.; Gruner, G. J. Electrochem. Soc. 2011, 158, A1-A5.

[109]Ge, J.; Cheng, G. H.; Chen, L. W. Nanoscale 2011, 3, 3084-3088.

[110]Liu, J. L.; Sun, J.; Gao, L. Nanoscale 2011, 3, 3616-3619.

[111]Huang, J. E.; Li, X. H.; Xu, J. C.; Li, H. L. Carbon 2003, 41, 2731-2736.

[112]Wu, T. M.; Lin, Y. W.; Liao, C. S. Carbon 2005, 43, 734-740.

[113]Lee, J. A.; Shin, M. K.; Kim, S. H.; Kim, S. J.; Spinks, G. M.; Wallace, G. G.; Ovalle-Robles, R.; Lima, M. D.; Kozlov, M. E.; Baughman, R. H. ACS Nano 2012, 6, 327-334.

[114] Yuan, L. Y.; Lu, X. H.; Xiao, X.; Zhai, T.; Dai, J. J.; Zhang, F. C.; Hu, B.; Wang, X.; Gong, L.; Chen, J.; Hu, C. G.; Tong, Y. X.; Zhou, J.; Wang, Z. L. ACS Nano 2012, 6, 656-661.

[115]Peng, C.; Zhang, S. W.; Jewell, D.; Chen, G. Z. Progress in Natural Science 2008, 18, 777-788.

[116]Liao, Y. Z.; Zhang, C.; Zhang, Y.; Strong, V.; Tang, J. S.; Li, X. G.; Kalantar-zadeh, K.; Hoek, E. M. V.; Wang, K. L.; Kaner, R. B. Nano Lett. 2011, 11, 954-959.

[117]Wang, H. L.; Hao, Q. L.; Yang, X. J.; Lu, L. D.; Wang, X. Nanoscale 2010, 2, 2164-2170.

[118]Yan, X. B.; Tai, Z. X.; Chen, J. T.; Xue, Q. J. Nanoscale 2011, 3, 212-216.

[119]Fan, H. S.; Wang, H.; Zhao, N.; Zhang, X. L.; Xu, J. J. Mater. Chem. 2012, 22, 2774-2780.

[120]Wu, Q.; Xu, Y. X.; Yao, Z. Y.; Liu, A. R.; Shi, G. Q. ACS Nano 2010, 4, 1963-1970.

[121]Niu, Z. Q.; Lu, P. S.; Shao, Q.; Dong, H. B.; Li, J. Z.; Chen, J.; Zhao, D.; Cai, L.; Zhou, W. Y.; Chen, X. D.; Xie, S. S. Energy Environ. Sci. 2012, 5, 8726-8733. 
[122]Wang, K.; Zhao, P.; Zhou, X. M.; Wu, H. P.; Wei, Z. X. J. Mater. Chem. 2011, 21, 16373-16378.

[123]Liu, Q. A.; Nayfeh, M. H.; Yau, S. T. J. Power Sources 2010, 195, 74807483.

[124]Antiohos, D.; Folkes, G.; Sherrell, P.; Ashraf, S.; Wallace, G. G.; Aitchison, P.; Harris, A. T.; Chen, J.; Minett, A. I. J. Mater. Chem. 2011, 21, 15987-15994.

[125]Zhang, L. L.; Zhao, S. Y.; Tian, X. N.; Zhao, X. S. Langmuir 2010, 26, 17624-17628.

[126]Xu, J. J.; Wang, K.; Zu, S. Z.; Han, B. H.; Wei, Z. X. ACS Nano 2010, 4, 5019-5026.

[127]Wang, D. W.; Li, F.; Zhao, J. P.; Ren, W. C.; Chen, Z. G.; Tan, J.; Wu, Z. S.; Gentle, I.; Lu, G. Q.; Cheng, H. M. ACS Nano 2009, 3, 1745-1752.

[128]Kumar, N. A.; Choi, H. J.; Shin, Y. R.; Chang, D. W.; Dai, L. M.; Baek, J. B. ACS Nano 2012, 6, 1715-1723.

[129]Zhang, K.; Zhang, L. L.; Zhao, X. S.; Wu, J. S. Chem. Mater. 2010, 22, 1392-1401.

[130]Liu, S.; Liu, X. H.; Li, Z. P.; Yang, S. R.; Wang, J. Q. New J Chem 2011, 35, 369-374.

[131]Lee, T.; Yun, T.; Park, B.; Sharma, B.; Song, H. K.; Kim, B. S. J. Mater. Chem. 2012, 22, 21092-21099.

[132]Biswas, S.; Drzal, L. T. Chem. Mater. 2010, 22, 5667-5671.

[133]Davies, A.; Audette, P.; Farrow, B.; Hassan, F.; Chen, Z. W.; Choi, J. Y.; Yu, A. P. J. Phys. Chem. C 2011, 115, 17612-17620.

[134]Kim, K. S.; Park, S. J. Electrochim. Acta 2011, 56, 1629-1635.

[135]Lu, X. J.; Dou, H.; Yuan, C. Z.; Yang, S. D.; Hao, L.; Zhang, F.; Shen, L. F.; Zhang, L. J.; Zhang, X. G. J. Power Sources 2012, 197, 319-324.

[136]Kim, J. H.; Lee, K. H.; Overzet, L. J.; Lee, G. S. Nano Lett. 2011, 11, 2611-2617.

[137]Hu, L. B.; Chen, W.; Xie, X.; Liu, N. A.; Yang, Y.; Wu, H.; Yao, Y.; Pasta, M.; Alshareef, H. N.; Cui, Y. ACS Nano 2011, 5, 8904-8913.

[138]Chen, W.; Rakhi, R. B.; Hu, L. B.; Xie, X.; Cui, Y.; Alshareef, H. N. Nano Lett. 2011, 11, 5165-5172.

[139]Kang, Y. J.; Kim, B.; Chung, H.; Kim, W. Synth. Met. 2010, 160, 25102514.

[140]Lv, P.; Zhang, P.; Feng, Y. Y.; Li, Y.; Feng, W. Electrochim. Acta 2012, 78, 515-523.

[141]Chen, P. C.; Chen, H. T.; Qiu, J.; Zhou, C. W. Nano Research 2010, 3 , 594-603.

[142]Chen, P. C.; Shen, G.; Sukcharoenchoke, S.; Zhou, C. Appl. Phys. Lett. 2009, 94, 233104.

[143] Chen, P. C.; Shen, G. Z.; Shi, Y.; Chen, H. T.; Zhou, C. W. ACS Nano 2010, 4, 4403-4411. 
[144]Boukhalfa, S.; Evanoff, K.; Yushin, G. Energy Environ. Sci. 2012, 5, 6872-6879.

[145]Chien, C. J.; Deora, S. S.; Chang, P. C.; Li, D. D.; Lu, J. G. Ieee T Nanotechnol 2011, 10, 706-709.

[146]Choi, B. G.; Yang, M.; Hong, W. H.; Choi, J. W.; Huh, Y. S. ACS Nano 2012, 6, 4020-4028.

[147]Li, Z. P.; Mi, Y. J.; Liu, X. H.; Liu, S.; Yang, S. R.; Wang, J. Q. J. Mater. Chem. 2011, 21, 14706-14711.

[148]Yu, G. H.; Hu, L. B.; Vosgueritchian, M.; Wang, H. L.; Xie, X.; McDonough, J. R.; Cui, X.; Cui, Y.; Bao, Z. N. Nano Lett. 2011, 11, $2905-$ 2911.

[149]Yu, G. H.; Hu, L. B.; Liu, N. A.; Wang, H. L.; Vosgueritchian, M.; Yang, Y.; Cui, Y.; Bao, Z. N. Nano Lett. 2011, 11, 4438-4442.

[150] Yuan, C. Z.; Yang, L.; Hou, L. R.; Li, J. Y.; Sun, Y. X.; Zhang, X. G.; Shen, L. F.; Lu, X. J.; Xiong, S. L.; Lou, X. W. Adv. Funct. Mater. 2012, $22,2560-2566$. 


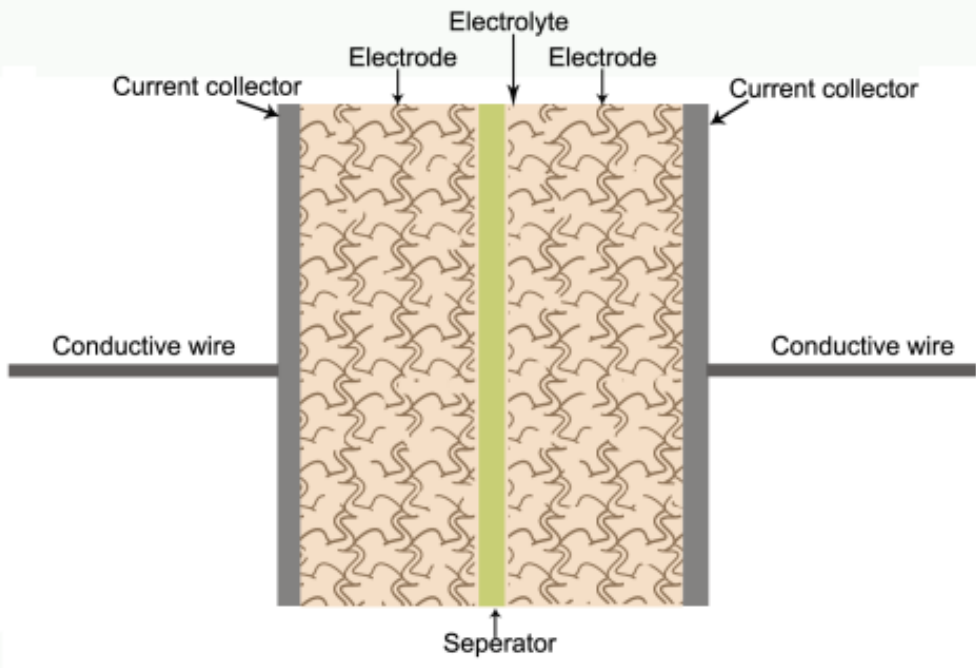

Figure 1 Schematic diagram of conventional SC 


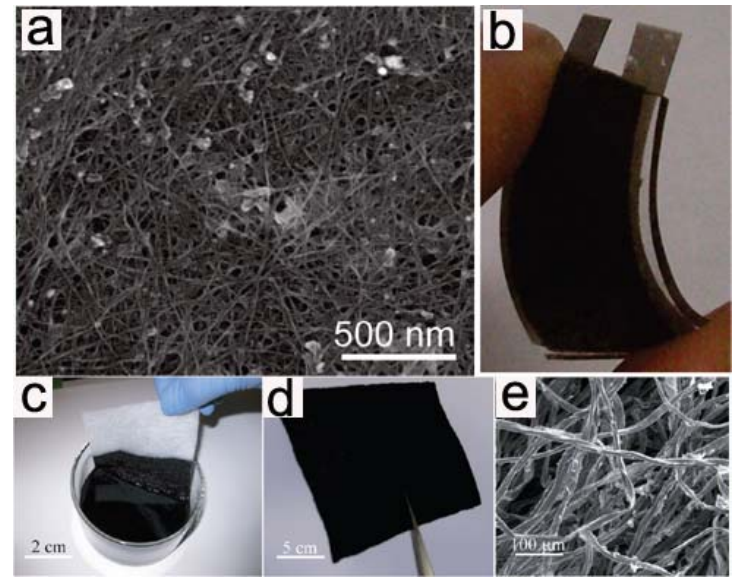

Figure 2. (a) Scanning electron microscopy image of as-deposited SWCNT networks. (b) Thin film SC using sprayed SWCNT films on PET as electrodes and a PVA/H3PO4 based polymer electrolyte as both electrolyte and separator. Reproduced with permission from reference [6]. Copyright 2009 American Chemical Society. (c) Conductive textiles are fabricated by dipping textile into an aqueous SWNT ink followed by drying in oven at $120{ }^{\circ} \mathrm{C}$ for $10 \mathrm{~min}$. (d) A thin, $10 \mathrm{~cm} \times 10 \mathrm{~cm}$ textile conductor based on a fabric sheet with $100 \%$ cotton and Rs of $4 \Omega$ /square. (e) SEM image of coated cotton reveals the macroporous structure of the cotton sheet coated with SWNTs on the cotton fiber surface. Reproduced with permission from reference [11]. Copyright 2010 American Chemical Society. 


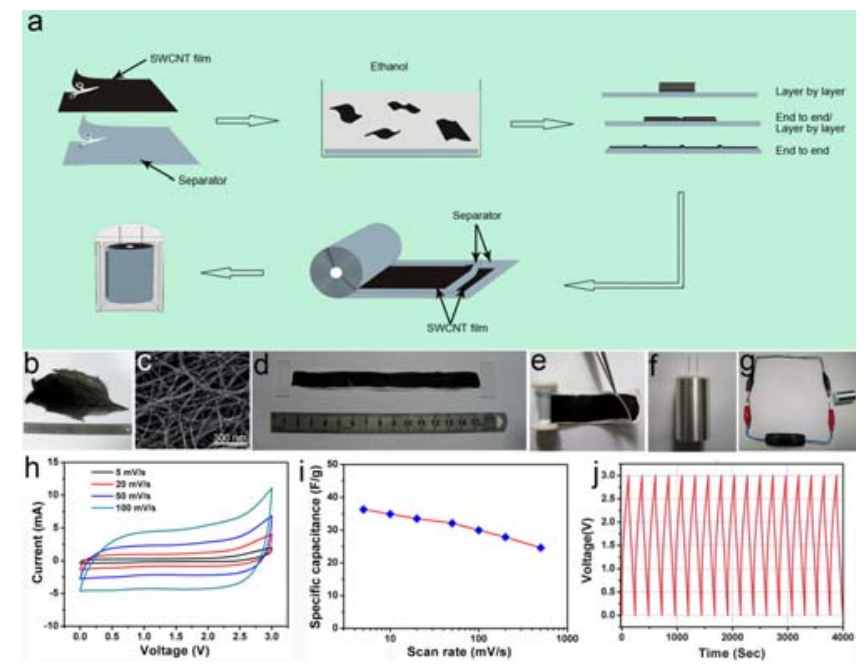

Figure 3 (a) Schematic diagram of assembling roll-designed SC using freestanding flexible SWCNT films. Optical image (b) and SEM image (c) of the directly grown SWCNT film. Optical image of (d) the SWCNT films spread out on a separator, (e) rolled design of the separator with SWNCT films and (f) the resulting roll-designed SC. (g) Optical image of the resulting roll-designed SC used to glow a red light-emitting diode (LED). (h) Typical CVs of a representative roll-designed SWCNT film SC at different scan rates. (i) The specific capacitance of the SWCNT film supercapacotor as a function of the scan rate. (j) Typical galvanostatic charge/discharge curves of the roll-designed SWCNT film SC. Reproduced with permission from reference [16]. Copyright 2011 Royal Society of Chemistry. 


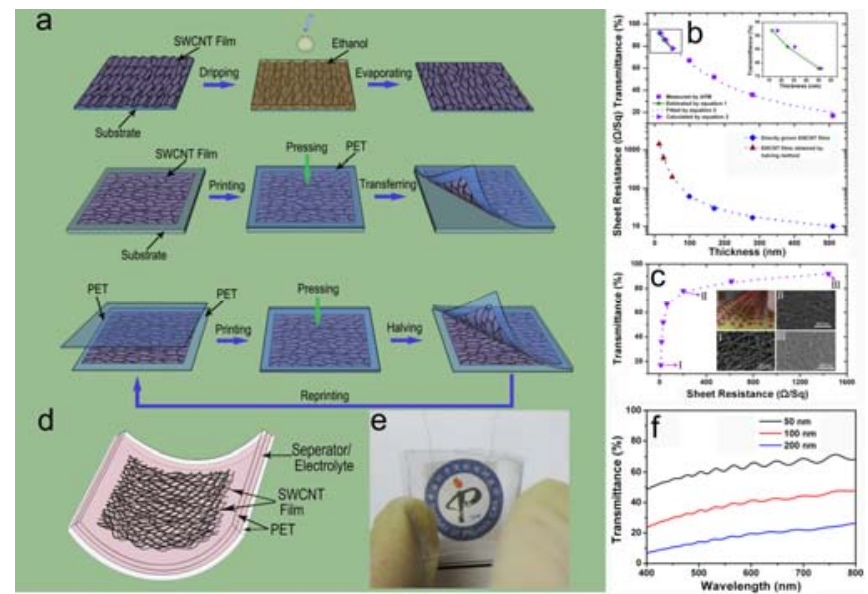

Figure 4. (a) Schematic diagram of preparing ultrathin SWCNT films by "repeated halving" approach: 1) spreading out a SWCNT film on to a substrate; 2) transferring SWCNT film from a substrate to PET substrate and 3) thinning thick SWCNT film to ultrathin SWNCT films by "repeated halving" approach. (b) Typical transmittance at $550 \mathrm{~nm}$ and sheet resistance curves of film as a function of SWCNT film thickness. Inset is the calculated thickness of halved thin SWCNT films by equation 1 and 2 as a function of transmittance at $550 \mathrm{~nm}$. (c) Typical sheet transmittance-resistance curve. Insets are the SEM and optical images of the SWCNT films with different thicknesses on PET substrates (I: $\sim 500 \mathrm{~nm}$, II: $\sim 50 \mathrm{~nm}$, and III: $\sim 13 \mathrm{~nm}$ ). (d) The schematic diagram of the transparent SWNCT film SC. (e) The optical image of the transparent SWCNT film SC. (f) Transmittance curve of SCs based on SWCNT films with different thickness. Reproduced with permission from reference [17]. Copyright 2012 Wiley-VCH. 


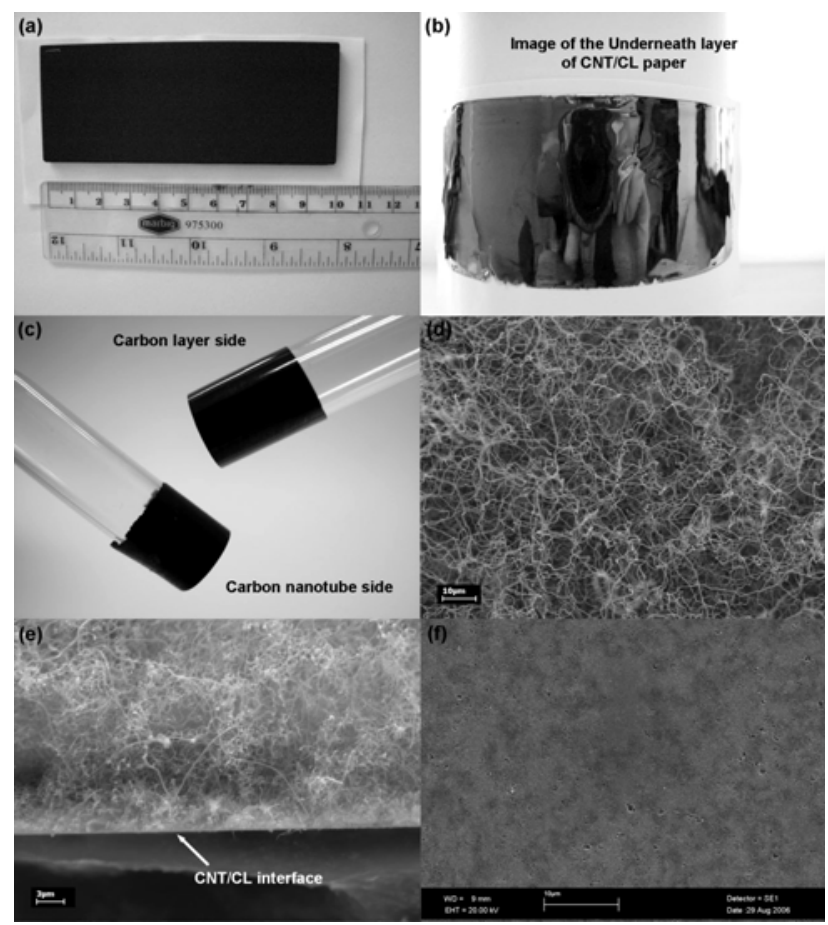

Figure 5. Scanning electron microscopy (SEM) and optical images of freestanding CNT/CL paper. a) Image of the upper surface of CNT/CL as grown on a $40 \mathrm{~cm} 2$ quartz plate by a CVD process. b) Image of the underneath surface of the carbon layer when removed from the substrate. As is obvious from the image, this layer is highly reflective. c) Image of CNT/CL paper removed from the quartz substrate and rolled onto glass rods, indicating the flexibility and mechanical robustness of both sides of the CNT/CL composite paper. d) SEM image of the top surface of the film, showing a dense entanglement of CNTs. e) SEM image of the cross section of the CNT/CL paper, showing an obvious 'intersection' (indicated by the white arrow) between the carbon layer and the upper CNT network layer. f) SEM image of the underneath of the CL layer, 
showing densely packed but still porous morphology. Reproduced with permission from reference [18]. Copyright 2008 Wiley-VCH.
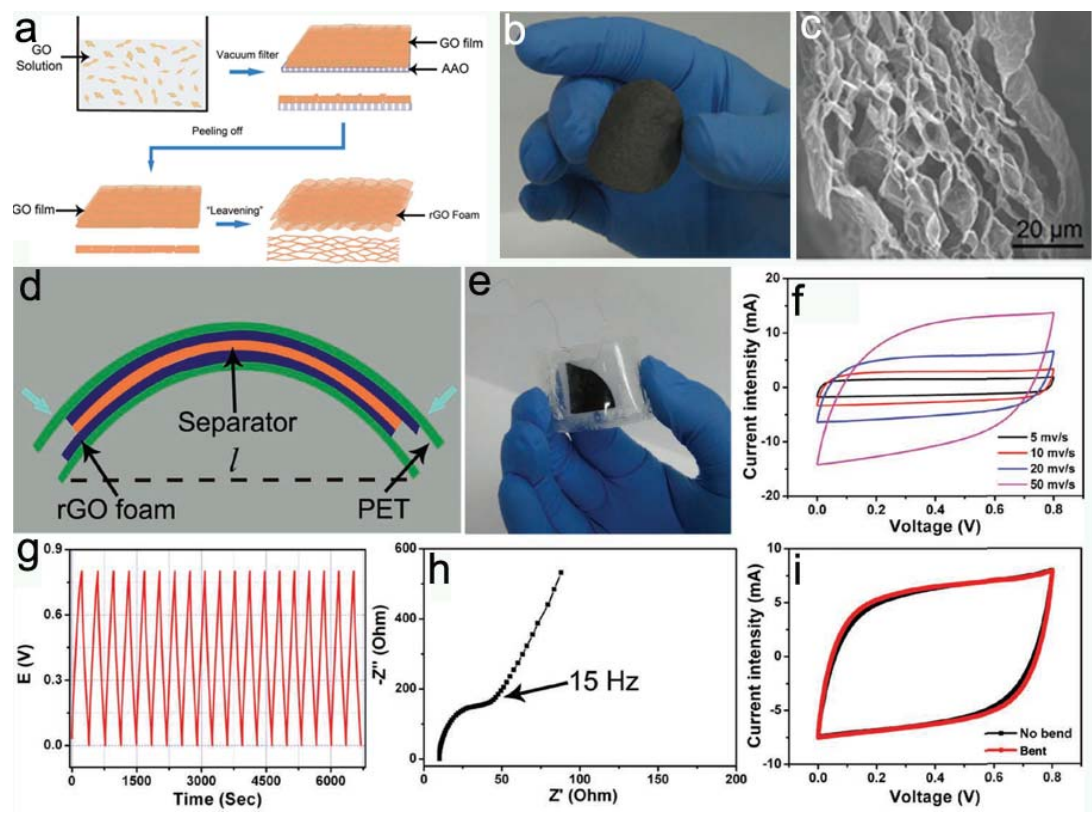

Figure 6 (a) Schematic drawings illustrating the leavening process to prepare rGO foams and (b) the photographs of free-standing paper-like rGO foams obtained by leavening method. (c) The cross-sectional SEM images of rGO foams formed at $90{ }^{\circ} \mathrm{C}$ for 10 hours in the autoclave with hydrazine monohydrate of $40 \mu \mathrm{L}$. (d) Schematic diagram of the flexible rGO foam SP, the distance $(l)$ between two sides of the rGO foam SP characterize its bending degree, (e) optical image of the flexible rGO foam SP, (f) CV curves of a representative $\mathrm{rGO}$ foam SP at different scan rates, (g) charge and discharge curve of rGO foam SP at the current of $0.5 \mathrm{~A} / \mathrm{g}$, (h) the Nyquist impedance plot of the rGO foam SP with frequency ranging from $10 \mathrm{kHz}$ to $1 \mathrm{~Hz}$, and (i) the CV of the rGO foam SP before $(l=3 \mathrm{~cm})$ and after $(l=2 \mathrm{~cm})$ bending. Reproduced with permission from reference [83]. Copyright 2012 Royal Society of

Chemistry. 

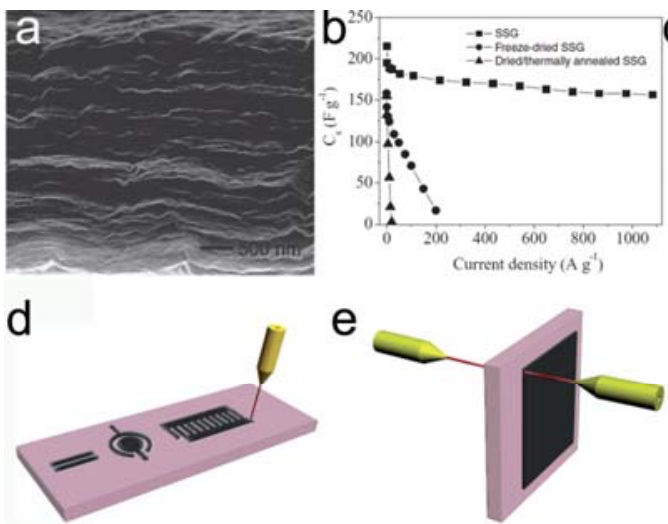

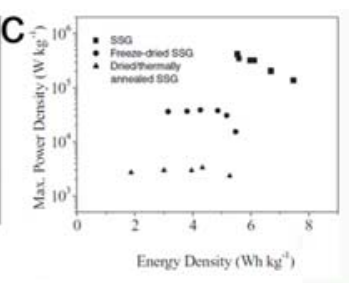

g

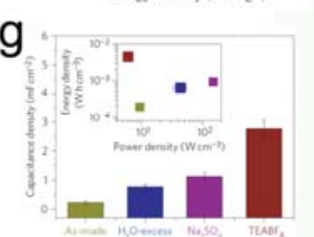

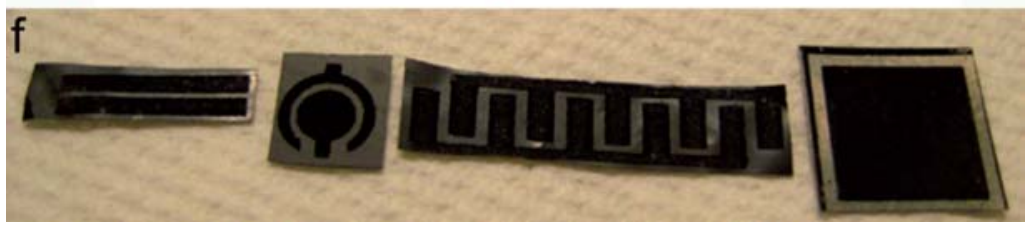

Figure 7 (a) SEM inmage of the solvated graphene film. (b) Gravimetric capacitances measured at various charge/discharge currents. (c) Ragone plots of the solvated graphene films-based SPs. The energy density and maximum peak power density are against the mass of the active materials in the electrodes. All the tests with a $1 \mathrm{~V}$ operation voltage were carried out in $1.0 \mathrm{M}$ aqueous $\mathrm{H}_{2} \mathrm{SO}_{4}$ electrolyte. Reproduced with permission from reference [84]. Copyright 2011 Wiley-VCH. Schematics of $\mathrm{CO}_{2}$ laser-patterning of free-standing hydrated $\mathrm{GO}$ films to fabricate RGO-GO-RGO devices with in-plane (d) and sandwich geometries (e). The black contrast in the top schematics corresponds to RGO, and the light contrast to unmodified hydrated GO. (f) Photographs of patterned films with different styles. (g) Histogram comparison of area-based capacitance density of a sandwich device in different electrlyte. Inset: volumetric energy density versus power density data of the corresponding devices. (h) Schematic illustration of the fabrication of laser-scribed graphene-based electrochemical capacitors. (i) Energy and power densities of LSG-Ecs compared with commercially available AC-EC, aluminum electrolytic capacitors, and a lithium thin-film battery. Reproduced with permission from reference [87]. Copyright 2011 Nature Publishing Group. 

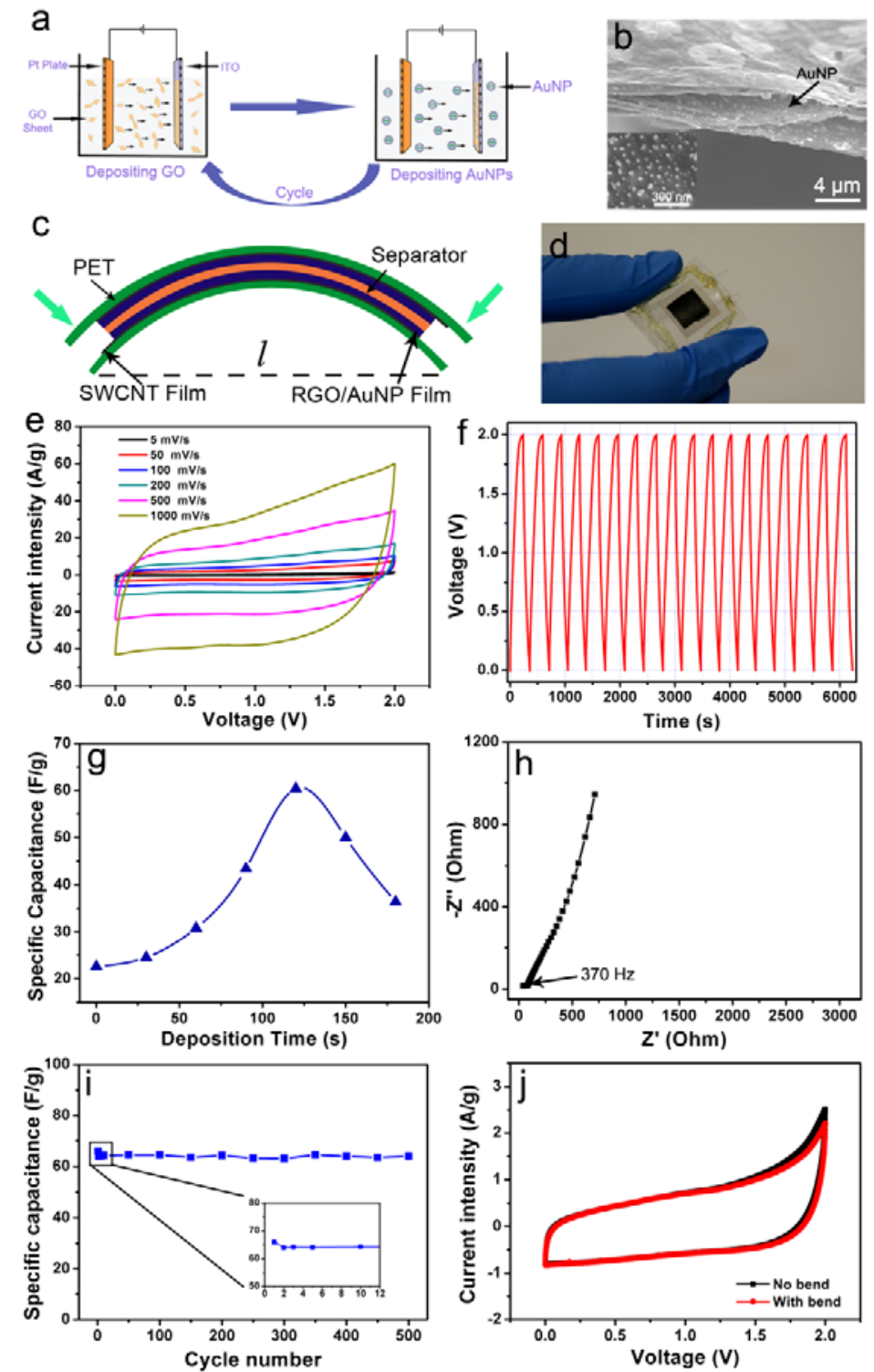

Figure 8 (a) Schematic process of constructing alternately multilayered GO/AuNP films on ITO surface cell. These two steps are the basic buildup 
sequence for the architecture of the multilayered $\mathrm{GO} / \mathrm{AuNP}$ composite films. (b) The cross-section SEM image of multilayered RGO/AuNP hybrid film. Inset is the SEM image of AuNPs on the graphene sheet surfaceThe schematic diagram (c) and optical image (d) of the flexible multilayered RGO/AuNP hybrid film SP using SWCNT film as current collector. (e) The CV curves of a representative RGO/AuNP hybrid film (AuNP deposition time: 120s) SP at different scan rates. (f) Typical galvanostatic charge/discharge curves of the representative multilayered hybrid film SP at $1 \mathrm{~A} \mathrm{~g}^{-1}$. (g) Specific capacitance of RGO/AuNP film SPs as a function of AuNP deposition time. (h) The Nyquist impedance plot of the hybrid film SP with frequency ranging from $100 \mathrm{kHz}$ to $1 \mathrm{~Hz}$. (i) Variation of capacitance stability of the hybrid film SP with cycle number. (h) The CV of the hybrid film SP before $(l=3 \mathrm{~cm})$ and after $(l=2 \mathrm{~cm})$ bending. 

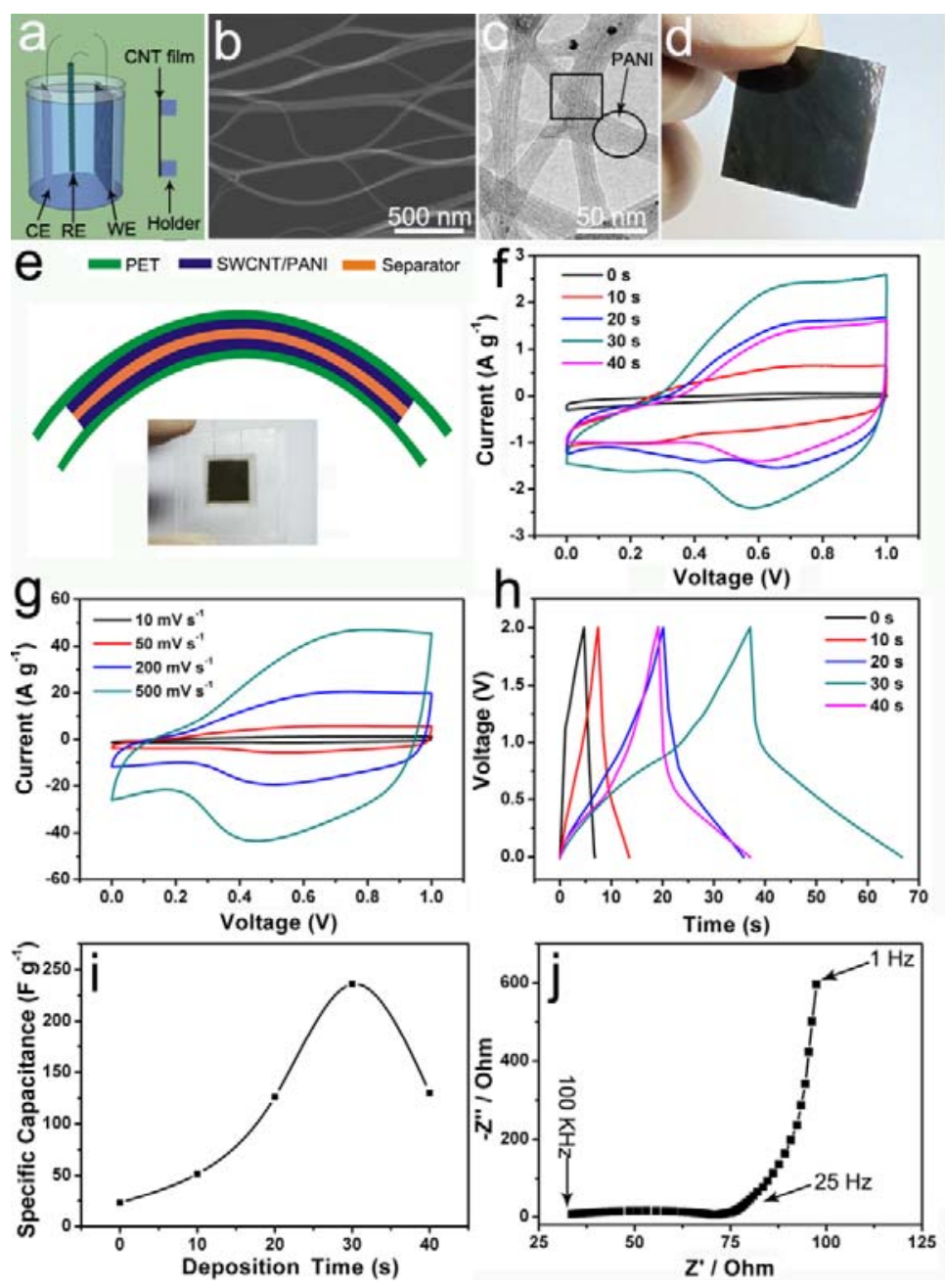

Figure 9 (a) Sketch of PANI electrodeposition using the directly grown SWCNT film as template. (CE: counter electrode, RE: reference electrode, WE: working electrodes) ((a) Schematic diagram and optical image of a flexible SWCNT/PANI hybrid film SP. (b) CV curves of SPs based on SWCNT/PANI hybrid film under different PANI deposition time, scan rate: 20 $\mathrm{mV} / \mathrm{s}$. (c) CVs of SWCNT/PANI hybrid film SPs at different scan rates. (d) 
Charge/discharge curves and (e) specific capacitance of SPs based on SWCNT/PANI hybrid film under different PANI deposition time. (f) Nyquist impedance plots of the SWCNT/PANI hybrid film SP with frequency ranging from $100 \mathrm{kHz}$ to $1 \mathrm{~Hz}$. Reproduced with permission from reference [91]. Copyright 2012 Wiley-VCH. 

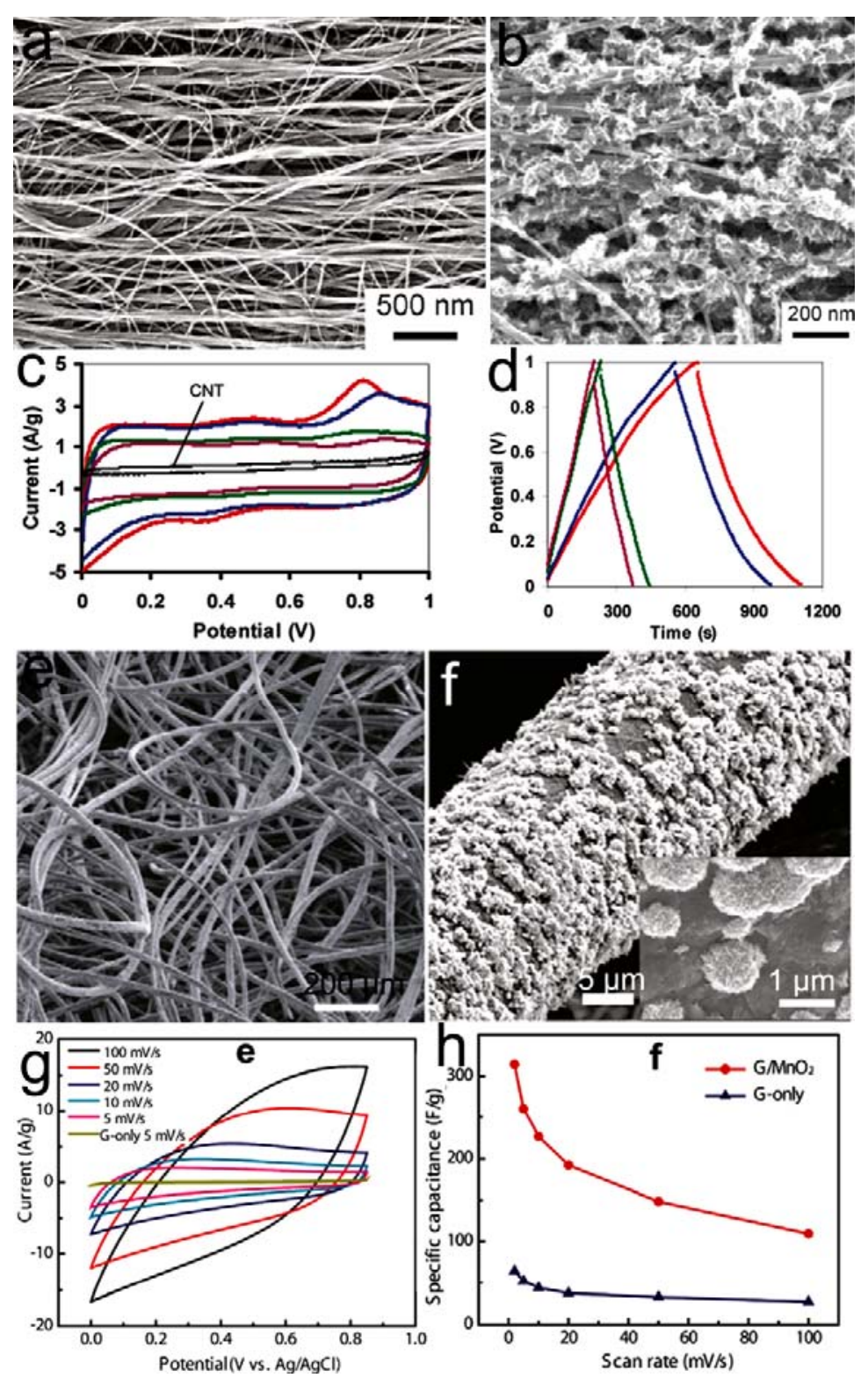
Figure 10 (a) CNT alignment in the electrode. (b) Morphology and microstructures of CNT sheet/ $\mathrm{MnO}_{\mathrm{x}}$ composites deposited for $5 \mathrm{~s}$. (c) CVs of the composites deposited for 15 (red), 30 (blue), 60 (green), and $180 \mathrm{~s}$ (brown) at 5 $\mathrm{mV} / \mathrm{s}$. (d) The charge/discharge curves for the composites at $1 \mathrm{~A} / \mathrm{g}$. Reproduced with permission from reference [136].Copyright 2010 American Chemical Society. (e) SEM image of a sheet of graphene-coated textile after $60 \mathrm{~min}$ $\mathrm{MnO}_{2}$. (f) SEM image of a typical microfiber with conformal coating of $\mathrm{MnO}_{2}$ nanostructures in the same textile. (Inset) High magnification SEM image showing the nanoflower structure of electrodeposited $\mathrm{MnO}_{2}$ particles and a clear interface between $\mathrm{MnO}_{2}$ nanoflower and underneath graphene nanosheets. $(\mathrm{g})$ $\mathrm{CVs}$ for graphene/MnO2-textile electrode at different scan rates in $0.5 \mathrm{M}$ aqueous $\mathrm{Na}_{2} \mathrm{SO}_{4}$ electrolyte ( $\mathrm{CV}$ curve for graphene-only textile at a scan rate of $5 \mathrm{mV} / \mathrm{s}$ included for comparison). (h) Comparison of specific capacitance values between graphene/ $\mathrm{MnO}_{2}$-textile and graphene nanosheets-only textile at different scan rates. Reproduced with permission from reference [148].Copyright 2010 American Chemical Society. 